\title{
An evaluation of dual-process theories of reasoning
}

\author{
MAGDA OSMAN \\ University College London, London, England
}

\begin{abstract}
Current theories propose that reasoning comprises two underlying systems (Evans \& Over, 1996; Sloman, 1996; Stanovich \& West, 2000). The systems are identified as having functionally distinct roles, differ according to the type of information encoded, vary according to the level of expressible knowledge, and result in different responses. This article evaluates the arguments and the evidence from a select number of key tasks that have been supportive of dual-reasoning theorists' proposals. The review contrasts the dualist approach with a single-system framework that conjectures that different types of reasoning arise through the graded properties of the representations that are utilized while reasoning, and the different functional roles that consciousness has in cognition. The article concludes by arguing in favor of the alternative framework, which attempts to unify the different forms of reasoning identified by dual-process theorists under a single system.
\end{abstract}

Traditionally, logical thinking and intuition have been viewed as rival modes of thought. The former is deliberate, achieving accurate and justifiable representations of the world, and the latter is ineffable, producing best-guess answers to problems without any discernable effort. This rivalry, summarized by Hammond (1996) as a distinction between intuitive and analytical thinking, has been the basis for much psychological investigation in the study of reasoning. Recently, in their theoretical frameworks many theorists have appealed to a similar distinction proposing that reasoning comprises two underlying systems that serve functionally separate roles (Cosmides \& Tooby, 2000; Epstein, 1973; Evans \& Over, 1996; Goel, 1995; Kahneman \& Frederick, 2002; Reyna \& Brainerd, 1990; Shafir \& LeBoeuf, 2002; Sloman, 1996; Stanovich \& West, 2000).

The aim of this article is to examine three key issues that have arisen in the advent of dual-process accounts of reasoning: What are the main distinctions between the two reasoning systems? What evidence is used to support this view? Can a single-system framework accommodate this evidence? To address these points, the review begins by briefly outlining the development of the dualist perspective and introducing in detail three of the main dual-reasoning theories: Evans and Over's (1996)

The support of the Economic and Social Research Council (ESRC) is gratefully acknowledged. The work was part of the program of the ESRC Research Centre for Economic Learning and Social Evolution. I thank Axel Cleeremans, Darrell Collins, Celia Heyes, David Lagnado, Ben Newell, Maxwell Roberts, Matthew Saxton, and David Shanks for advice and guidance, and also Jonathan Evans, David Over, Brian Ross, and Steven Sloman for helpful comments on an earlier draft of this article. Correspondence should be addressed to M. Osman, Department of Psychology, University College London, Gower Street, London WC1E 6BT, England (e-mail: m.osman@ucl.ac.uk). dual-process theory; Sloman's (1996) two-systems theory; and Stanovich and West's (2000) two-systems theory (dual-process theories, hereafter). These have been instrumental in developing current research paradigms to explore and examine the processes involved in all aspects of reasoning behavior (i.e., deductive and inductive reasoning, judgment and decision making, and problem solving). Next, a contrasting single-system framework is described. The origins of this framework are in the learning literature, and so the introduction to this account is situated therein, and then the empirical implications for reasoning are made. The third section focuses on three types of task that have provided supporting evidence for dual-reasoning processes. The final section summarizes the main findings and considers their implications for dual-process theories and a single-system framework.

\section{Development of Dual-Process Theories of Reasoning}

The psychological origins of the dualist distinction between rational and irrational thinking can be traced back to James (1890/1950) and Freud (1900/1953). Both claimed that reasoning takes the form of two different modes of thought. James regarded reasoning as an experiential associative type of thinking, as well as a separate analytical deliberate mode. Similarly, Freud's dual theory of information processing distinguished between a primary system that is associative and unconscious, and a secondary system that is conscious and capable of rational thought. Epstein $(1973,1994)$ integrated aspects of Freud's psychodynamic account in his cognitive-experiential self-theory (CEST). The CEST was developed as a global theory of personality with two parallel systems. One is a nonexperiential information processor that is rational, affect-free, abstract, and analytical, and the second is an experiential information processor that is emotionally 
driven, encoding experiences in the form of concrete exemplars. The theory claims that the two systems developed to fulfill different functional roles and in turn have distinct evolutionary histories. The experiential system is the older of the two, operating in humans and nonhumans, and although crude, it processes information effortlessly. The analytic system has a relatively short evolutionary history, is unique to humans, and was developed to operate in the medium of language.

Early research on human judgment and decision making provided much evidence to suggest that there were competing modes of thought, one of which is intuitive and the other extensional, and that they led to entirely different types of responses (e.g., Hammond \& Summers, 1972; Tversky \& Kahneman, 1983). Tversky and Kahneman (1974) claimed that intuitive type reasoning is informal and unstructured, and relies on heuristics that include such computations as similarity, representativeness (i.e., the assessment of the degree of correspondence between a sample and a population), and attributions of causality. Tversky and Kahneman shared Epstein's (1973, 1994) view that the information the system acts upon is content specific and affective, whereas extensional reasoning is controlled, slow, and deliberate and acts as a regulator monitoring intuitive responses that it can choose to endorse or override.

Early dual-process theories led to a diverse set of assumptions about the nature and origins of the different types of reasoning identified. While the research programs developed from them center on different aspects of human behavior, the basic claims made are the same. The basis for proposing separate reasoning systems stems from the view that experiences are ordered very differently according to how they are used to reason about the world. Experiences are arranged such that they form initial intuitive understandings of the world that, when organized, can be used to control and manipulate certain mental activities. These two alternative uses result in differences in the way information is encoded, differences in behavior, and differences in expressible knowledge.

This article focuses on three of the most influential dualprocess theories currently pervading a wide range of work on thinking and reasoning. To begin, these theories will be described more thoroughly in terms of their characterization of the two processes, the functions they serve, and the type of information they encode. In the remainder of this section, connections are drawn across the theories and then discussed. Included in this section is the first of two appraisals of the dual-process account, to provide some evaluation of the actual theoretical proposals made. At the end of this article comes the second evaluation, in which the theories are reviewed in relation to empirical findings.

\section{Evans and Over's Dual-Process Theory}

Evans's $(1989,1996)$ heuristic-analytic theory provided the foundations for Evans and Over's (1996) current dual-process theory. Evans (1984) proposed that heuristic processes are preconscious, and their function is to select representations relevant to a particular problem space, in contrast to Tversky and Kahneman's (1974) characterization of heuristics as strategies that provide shortcuts to a solution. Analytic processes are conscious, which means broadly that they are a type of deliberate, explicit thinking. Their role is to operate on representations deemed relevant by heuristic processes that are then used to generate inferences and form judgments (e.g., Evans, 1995, 1996). They accomplish some forms of logical analysis but are essentially context dependent because they are based on the individual's experience.

Evans's (2002; Evans \& Over, 1996) current dualprocess account of reasoning incorporates his earlier proposals and is strongly influenced by the kinds of distinctions between cognitive systems set out by implicit learning theorists (Berry \& Dienes, 1993; A. S. Reber, 1993). This theory divides reasoning into two systems. System 1, which is essentially pragmatic, is based on prior experiences, beliefs, and background knowledge and achieves goals reliably and efficiently without necessarily accompanying awareness. It is characterized as implicit, associative, fast, and highly robust, and it is spared by both aging (Gilinsky \& Judd, 1994) and neurological damage (e.g., Deglin \& Kinsbourne, 1996). System 2 is explicit, sequential, controllable, and makes high demands of working memory. System 2 does not typically operate according to normative logical conventions, but it is capable of achieving solutions to logical problems as well as a range of problem types (e.g., hypothesis testing, hypothetical thinking, forecasting, and consequential decision making). Compared with System 1, System 2 is slow, but in compensation it affords flexibility and controllability.

Evans and Over (1996) make an important distinction between the function of System 1, which is domain specific, and its mechanism, which is domain general. The system is domain general in the sense that it is likened to a neural network where knowledge is expressed as activations of particular units in the network, and not contentspecific rules. The functioning is domain specific because the knowledge acquired under this system develops in highly localized domains. Evans and Over refer to Nisbett, Krantz, Jepson, and Kunda's (1983) study to illustrate this difference. Nisbett et al. presented problems based on the law of large numbers to participants without formal statistical knowledge. In one problem, participants were asked to choose which of a range of descriptions best explained why players showed better performance earlier rather than later in the sporting season. Those with experience in the domain gave a statistical explanation for changes in players' form, but inexperienced participants chose a determinate account. The difference in descriptions showed that the application of the law was based on domain-specific knowledge acquired through experience (i.e., based on exposure to sports matches). Moreover, the knowledge was likely to be implicit rather than explicit since participants did not have any formal understanding of the law itself and had acquired this knowledge incidentally. 
In Evans's (1984) heuristic-analytic account, he suggests that normative theories of rationality (e.g., logic, decision theory) may not provide appropriate methods of assessing human reasoning and often lead to the conclusion that we are irrational. However, if rationality is judged by the application of relevant knowledge to form inferences, humans are rational. This issue is expanded in Evans and Over's (1996) dual-process theory, leading to the proposal of two forms of rationality. One is personal (rationality ${ }_{1}$ ) and identifies reasoning behavior that is generally reliable and efficient for achieving one's goals. The other is impersonal (rationality ${ }_{2}$ ), a type of reasoning behavior that suggests acting when one has a rationale for what one does, and is sanctioned by a normative theory.

\section{Sloman's Dual-System Theory}

The focus of Sloman's (1996, 2002) dual-system theory is the computational distinction between two types of reasoning systems. System 1 is associative and is attuned to encoding and processing statistical regularities, frequencies, and correlations in the environment. System 2 is rule based. The representations in this system are symbolic and unbounded, in that they are based on propositions that can be combined to form larger and more complex sets of propositions.

Sloman (1996) uses Smolensky's (1988) connectionist framework to describe the computational differences between System 1 and System 2. Smolensky contrasted two types of inferential mechanisms within a connectionist framework: an intuitive processor and a conscious rule interpreter. Sloman claims that both System 1 (intuitive processor) and System 2 (conscious rule interpreter) are implemented by the same hardware but use different types of knowledge that are differently represented. The representations within System 1 are characterized as associative and are automatically generalizable, allowing for a fast inferential process. Unlike System 2, the representations of System 1 cannot be combined in novel ways, so that reasoning in System 1 is limited to what has already been represented.

System 2 is a high-level processor abstracting information and expressing knowledge as production rules (e.g., "if . . . then ..."), as described in J. R. Anderson's (1993) ACT-R (atomic components of thought) model. This knowledge is accessible, verbalizable, and shared, in that the rules that are formulated describe a task domain that conforms to predefined conventions or syntax that is relevant within a cultural community. Sloman (1996) uses chess to illustrate this. Players of chess have common knowledge of the rules, which have been sanctioned by a community more extensive than the two players. The rules refer to constituents of the game (i.e., the pieces and the moves) that can be expressed in terms of a common reference. In this sense, the rule interpreter involves reasoning about a set of moves at the same conceptual level as the concepts within the problem domain - namely, the game itself.
The two systems are fundamentally distinguished by the underlying computational mechanisms, not by whether they differentially involve implicit and explicit processing. In fact, Sloman's (1996) aligning of the two systems with implicit and explicit processes is tentative. He suggests that although the content of awareness may be used to separate the two systems, it is neither a necessary or sufficient way of doing so because, in his view, both System 1 and System 2 can occur without conscious awareness. The content of awareness reflects different conscious understandings of responses to a problem. Individuals using the associative system are conscious of the product of the computation - that is, the response, rather than the actual process used to achieve it. By contrast, the rulebased system generally occurs with full awareness of both the process and the result.

The relationship between the systems is described as interactive, and the degree to which they are each applied to a problem will vary from individual to individual, depending on the reasoner's skill, knowledge, and experience. Moreover, neither system has an exclusive problem domain to which they are applied. As Sloman (1996) suggests: "It may not even be possible because both Systems may contribute to a particular response" (p. 6). This forms an important basis for proposing a criterion to identify dissociations between the systems. Sloman (1996, 2002) claims that one way of examining differences between the systems is by identifying tasks that satisfy what he terms Criterion S. This refers to situations where individuals are led to respond in a manner consistent with System 1 but then come to realize, with or without external prompting, an alternative response consistent with System 2. System 1 remains influential despite its subsequent replacement by System 2 thinking because associative processes are highly salient. Sloman (1996) describes a host of studies reporting findings that conform to the conditions of Criterion S: for example, categorization tasks (e.g., Rips, 1989; Smith \& Sloman, 1994), judgment and decision making (e.g., Fiedler, 1988; Tversky \& Kahneman, 1983), conditional reasoning (e.g., Cheng \& Holyoak, 1985; Kirby, 1994), and syllogistic reasoning (e.g., Evans, Barston, \& Pollard, 1983).

In response to the question of why reasoning should be viewed as having two systems, Sloman (1996) suggests, "One answer is that the Systems serve complementary functions" (p. 18). Sloman claims that the systems operate in concert but are developed to suit different types of knowledge and result in different outcomes that are each useful in different ways. For example, disciplines such as mathematics and physics require a combination of intuitive thinking (System 1) and structured rule-based analysis (System 2).

\section{Stanovich and West's Two-Systems Theory}

Stanovich and West's $(2000,2002)$ two-systems theory of reasoning is primarily concerned with what causes differences in the way individuals reason. Individuals have been found to respond differently to reasoning tasks 
according to how they construe them (e.g., Osman \& Laming, 2001), their prior knowledge (e.g., Galotti, Baron, \& Sabini, 1986), the strategies they use (e.g., Klayman \& Ha, 1987), cognitive and personality styles (e.g., Stanovich \& West, 1998b, 1998c), and their ability (e.g., Guilford, 1959; Stanovich \& West, 1998b). Stanovich and West used this source of data as a way of examining human rationality. Essentially, they distinguish between three different accounts of individuals' departures from normative standards. One view is that individuals' poor performance on reasoning tasks reflects irrational tendencies (e.g., Nisbett \& Ross, 1980; Tversky \& Kahneman, 1974). However, others suggest that individuals fail to perform well because of superficial cognitive slips in attention, or memory lapses (e.g., Cohen, 1981). Finally, individuals' performance might be consistent with a different normative model (e.g., Koehler, 1996), or the normative model may simply be inappropriately applied to assess responses to a particular task (e.g., Hilton, 1995; Schwarz, 1996).

To address these issues, Stanovich and West (1998a, $1998 b, 1998 \mathrm{c}$ ) examined the magnitude of the correlation between performance on a range of tasks (deductive, inductive, statistical, decision making) and cognitive ability. They found that performance correlated with cognitive ability in every case, which suggested that computational limitations could be used as a predictor of participants' responses on reasoning and problem-solving tasks. The examination of individual differences motivated Stanovich and West (2000) to present a unifying framework proposing two functionally distinct reasoning systems.

System 1 is implemented automatically, is unconscious, and is context dependent, relying on the types of heuristics described by Evans (1989). System 2 is a controlled process that is purely analytical and is based on making abstractions that do not rely on context. The primacy of System 1 leads to what Stanovich (1999) terms the fundamental computational bias, which is the tendency to automatically contextualize problems. The bias prevents individuals from reasoning about a task according to its logical properties; instead, they rely on cues from its context, which are interpreted in relation to real-life situations.

Stanovich and West propose that the large differences in cognitive ability result from the two systems cuing different responses. They elaborate on this by describing two types of intelligence. One embodies analytic intelligence and is measured by psychometric tests (e.g., Scholastic Aptitude Tests [SAT]). The other is termed interactional intelligence (Levinson, 1995) and is pragmatic in nature. Individuals of higher cognitive ability are able to recruit analytical processes (System 2), avoiding the temptation to rely on simple general-purpose heuristics that would be unsuitable for a particular task. Conversely, individuals who perform less well are more likely to rely on heuristics readily available in System 1.
Of the three dual-process theories, that of Stanovich and West (2000) provides the most detailed explanation of how the two systems of reasoning developed, by outlining their evolutionary histories. System 1 serves evolutionary rationality; this type of rationality is designed to closely track increases in the reproduction probability of genes and identifies natural regularities in the environment. System 2 serves instrumental rationality: "System 2 , while also clearly an evolutionary product, is also primarily a control System focused on the interests of the whole person. It is the primary maximizer of an individual's personal utility" (Stanovich \& West, 2000, p. 656). This system is attuned to normative models and focuses on making abstractions.

On the issue of the evolutionary origins of reasoning, these distinctions position Stanovich and West (2000) apart from evolutionary psychologists (e.g., Fiddick, Cosmides, \& Tooby, 2000; Hertwig, Ortmann, \& Gigerenzer, 1997), who deny content-free logical processes as being part of the reasoning architecture. In Stanovich and West's (2000) view, many reasoning tasks trigger heuristics (e.g., conjunction problem, selection task) that make evolutionary sense (e.g., Hilton, 1995; Levinson, 1995; Stanovich \& West, 2000), but individuals using analytic processes adopt a normative construal of the task because they are motivated to maximize personal utility (e.g., Baron, 1985; Stanovich, 1999). The goals programmed to suit the genes' interests (i.e., the reproduction probability of genes) and the goals by which the organism operates (i.e., to maximize the individual's personal utility) are differentially expressed in Systems 1 and 2. In this sense, both systems are treated as optimally designed for different purposes and so produce different outcomes.

\section{Commonalities Among the Theories}

In what ways do the claims of these theories overlap? They share many common features both with each other and earlier theoretical accounts (e.g., Epstein, 1973; Tversky \& Kahneman, 1983). They characterize the two systems in broadly the same way, suggesting that System 1 is associative, automatic, and fast, and that System 2 is rule-based, deliberate, flexible, and slow. The systems are said to have an interactive relationship and have overlapping domains to which they apply, but they also generate conflicting responses, which Sloman (1996) and Stanovich (1999; Stanovich \& West, 2000) regard as support for the existence of separate reasoning systems. These theorists acknowledge the similarities in their proposals; however, there are three particular issues that Evans and Over (1996) and Stanovich and West (2000) stress in their theories.

Implicit and explicit processing. Evans and Over (1996) and Stanovich and West (2000) highlight the links between their theories and implicit learning theorists' (Berry \& Dienes, 1993; A. S. Reber, 1993) conceptions of implicit and explicit processing. Both theoretical do- 
mains converge in suggesting that implicit processing occurs incidentally and without concomitant awareness, whereas explicit processing is deliberate and always accompanied by awareness. In addition, similar views are shared in the two processes' differential relationship with the central executive. Stanovich and West (2000) and Evans and Over suggest that System 1 makes virtually no demands on the central executive, and so allows complex information processing to occur automatically (e.g., depth perception, face recognition, practiced motor behavior, intuition in problem solving and game playing, and language processing).

System 2 is bound to cognitive constraints, and so processing is slow. The dependency of System 2 on the central executive enables knowledge to be externalized and therefore reportable, which makes processing in this system explicit. According to Evans (2000), "Explicit processes also involve conscious control and can thus be modified by appropriate verbal instructions" (p. 6). Evans and Over (1996) claim that the advantage of conscious reflective thought is that it provides the flexibility and foresight that the implicit system cannot offer. Stanovich (1999; Stanovich \& West, 2000) frames the advantages differently. He proposes that one of the main purposes of System 2 is to decouple information from the context automatically supplied by System 1, which sometimes interferes with the depersonalized and decontextualized representation that might be necessary for processing by System 2.

Two types of rationality and the evolutionary origins of the two systems. Two further issues discussed in more detail by Evans and Over (1996) and Stanovich and West (2000) are the systems' relationship to conceptions of rationality, and their evolutionary origins. Evans and Over's two rationality types do not map directly onto the two reasoning systems but are described as loosely linked to them. Their distinction is intended to account for what they identify as an "apparent paradox" in reasoning that appears on the one hand to be irrational and fraught with biases and fallacies, and yet, on the other hand, is clearly adapted to function well in everyday life. In their discussion on the evolutionary basis of the two systems, Over and Evans (1997) claim that explicit thinking is "a uniquely human facility added late in evolutionary terms to a biologically tacit System, which is similar to that operative in other animals" (p. 263). Implicit processes are said to originate from three sources: innate modules, implicit learning, and automation of knowledge that was first acquired explicitly. Over and Evans argue that System 2 is a prerequisite for rational ${ }_{2}$ thought, which is unique to humans, whereas rationality ${ }_{1}$ is instantiated by System 1, which is shaped both by past successes of an evolutionary nature and through the experiences acquired over the course of an individual's lifetime.

Stanovich and West's (2000) views on rationality are interlinked with their claims on the evolutionary basis of the two systems. They distinguish between two forms of rationality: normative and evolutionary. Normative ra- tionality is broadly defined as the maximization of the goals of the individual organism. Evolutionary rationality is defined in relation to Dawkins's (1976) view of adaptive optimization at the level of the gene. Unlike Evans and Over (1996), Stanovich and West (2000) propose a one-to-one mapping between the two types of rationality and Systems 1 and 2.

\section{Appraisal of Dual-Process Theories}

How does a single-system perspective contrast the claims made by dual-process theorists? This section is concluded by a discussion on reasoning theorists' (e.g., Bucciarelli, 2000; Hammond, 1996; Margolis, 1987; Moshman, 2000; Newstead, 2000; Oberauer, 2000; Roberts, 1997; Stevenson, 1997) challenges to the dualist framework of reasoning, who in turn propose a singlesystem account. The aim here is to examine these two positions, forming the basis for introducing the alternative single-system framework in the next section.

One of the principal arguments made against dualprocess theories is that the proposed dichotomy does not adequately accommodate the range of processes identified in studies of reasoning. Moshman (2000) argues that the differences outlined between implicit heuristic reasoning and explicit analytical reasoning (e.g., Evans \& Over, 1996; Stanovich \& West, 2000) confound two orthogonal distinctions - namely, those between implicit and explicit processing and between heuristic and analytic processing. Evidence from studies of logical and mathematical reasoning (e.g., Braine \& O'Brien, 1998; Dixon \& Moore, 1997; Hawkins, Pea, Glick, \& Scribner, 1984) shows that inferences consistent with normative principles are made automatically and that explicit reasoning can also involve the deliberate discovery and application of heuristics (e.g., Kuhn, 2000; Moshman, 1999). Moshman (2000) proposes that a multiple-systems framework should include four possible types of processing: implicit heuristic processing, implicit rule-based processing, explicit heuristic processing, and explicit rulebased processing.

Stevenson (1997) claims that criteria used to identify the two reasoning systems do not accommodate examples of implicit and explicit processing that involve the acquisition and application of simple and complex rules (e.g., Dienes \& Fahey, 1998; Hayes \& Broadbent, 1988). Stevenson suggests that implicit processing involves the encoding and application of fragmentary knowledge and is situated at one end of a continuum of reasoning behavior (Burns \& Vollmeyer, 2002; Geddes \& Stevenson, 1997). Explicit processing generates organized representations of the current state of affairs that can be manipulated and combined with prior instances to form new representations and is located at the other end of the continuum (Stevenson, 1997). Between these poles, representations of knowledge vary according to their accuracy and relevance to the current problem space.

Hammond's (1996) cognitive continuum theory proposes that different forms of cognition (intuitive, analyt- 
ical, common sense) are situated in relation to one another along a continuum that places intuitive processing at one end and analytical processing at the other. The properties of reasoning (e.g., cognitive control, awareness of cognitive ability, speed of cognitive activity) vary in degree, and the structural features of the tasks that invoke reasoning processes also vary along the continuum, according to the degree of cognitive activity they are predicted to induce (Dunwoody, Haarbauer, Mahan, Marino, \& Tang, 2000). Hammond claims that the advantage of his approach, as an alternative to a dichotomous one, is that it is parsimonious because it accommodates a broader range of processing within a single-system framework.

The question of whether dual-cognitive systems can adequately capture the full range of processing types has been raised in response to dual theories of learning and memory (e.g., Block, 1995; Cleeremans, 1997; Farah, 1994; O’Brien \& Opie, 1999). The proposal that there are separable learning systems has been challenged (e.g., Meier \& Perrig, 2000; Perruchet \& Baveux, 1989; Shanks \& Channon, 2002; Shanks \& Johnstone, 1999), as well as the methodologies used to investigate the claimed dichotomy (see, e.g., Dulany, 1991; Holender, 1986; Shanks \& St. John, 1994; Velmans, 1991). In response to this, many propose (e.g., Cleeremans, 1997; Cleeremans \& Jiménez, 2002; Farah, 1994; O’Brien \& Opie, 1999) a single-system learning framework incorporating a continuum between implicit and explicit processes. This position is particularly relevant because some dual theorists (Evans \& Over, 1996; Stanovich \& West, 2000) emphasize the relationship between their dichotomy and that proposed by implicit learning theorists (e.g., Berry \& Dienes, 1993; A. S. Reber, 1989). It also provides the platform for which to discuss the different theoretical claims that are made in the learning literature, and it establishes the foundations by which the proposed alterative framework can be discussed, first in terms of its applications to learning and then to reasoning. The next section focuses on Cleeremans and Jiménez's (2002) framework, which will be used as a basis for contrasting and evaluating the dualist reasoning approach.

\section{The Alternative Framework}

The following discussion first describes the proposals of Cleeremans and Jiménez's (2002) dynamic graded continuum (hereafter DGC) framework. The aim here is not to advance a new theory of reasoning but to provide a framework that can be used to assess dual-process theories and the evidence used to support their claims. The first part of this section outlines the DGC framework in relation to its origins, and then shows how it extends to reasoning. The latter part of the section then discusses the differences between the claims it makes, in contrast to dual-process theories of reasoning.

The DGC framework proposes that the quality of representation (i.e., strength, distinctiveness, stability) lies along a continuum and the increase in the quality of the representation along the continuum leads to a correspond- ing progression in the type of learning, from implicit, to explicit, to automatic. For each form of learning, consciousness has a different functional role (Cleeremans, Destrebecqz, \& Boyer, 1998).

Cleeremans and Jiménez (2002) characterize consciousness as dynamic because it changes its states and dispositions (e.g., Cleeremans, 1997; Perruchet \& Vinter, 2002). Rather than being an all-or-nothing state, it is viewed as graded according to different types of subjective experience (e.g., Farah, 1994; Mathis \& Mozer, 1996). Both of these characteristics lead to different functional roles in learned behavior. To illustrate, learning to cross the road is first achieved by explicit instructions to look left and right in order to anticipate where and when oncoming traffic will appear; this requires active conscious control of the behavior. Eventually, the steps involved in crossing the road become practiced and habitual, and the learned behavior is carried out automatically, without the need for conscious control. The subjective experience of crossing the road changes as does the control that is executed over the corresponding behavior.

Cleeremans and Jiménez (2002) use a connectionist paradigm to describe the graded nature of representations. The cognitive system is viewed as a large set of interconnected processing modules organized hierarchically, and as such, representations are dynamic because they are transient patterns of activation over the units of each module (Rumelhart \& McClelland, 1986). Representations allow the cognitive system to monitor the intermediate results of processing (J. R. Anderson, 1995; KarmiloffSmith, 1986), and for representations to enter awareness they need to be active enough to gain strength, stability in time, and be sufficiently distinctive (e.g., Farah, 1994; O’Brien \& Opie, 1999; O'Reilly \& Munakata, 2000). These three properties define the "quality" of the representations on which the continuum is based.

Strength is defined as the amount and the level of activation of processing units. To illustrate, studies of complex implicit rule learning (e.g., Berry, 1991; Dienes \& Fahey, 1998) show that when learning is active and deliberate compared with nondirective, passive learning (e.g., observational learning), there is greater retention and control of the representations acquired, leading to transfer to new task domains. Recall and transfer tests are often used as ways of measuring the extent to which representations are strong enough to be retained and reliably called up by the central executive. Stability is the length of time a representation remains active during processing. Subliminal perception studies (e.g., Cheesman \& Merikle, 1984; Kunimoto, Miller, \& Pashler, 2001) show that subjective experience varies (e.g., random guesses to accurate estimates of detection of items presented) depending on the length of time that items are presented. As the duration of the items displayed decrease, so do participants' subjective experience and confidence in making accurate judgments about what they have been shown. Distinctiveness refers to the discriminability of representations. This can be measured according to the accuracy with which an in- 
dividual can recognize fragments of knowledge acquired through training. Studies of implicit memory (e.g., Crabb \& Dark, 1999; MacDonald \& MacLeod, 1998) have shown that accuracy in discriminating between old, learned items and new items is dependent on how well attended the material is at the time of encoding.

Farah's (e.g., Farah, Monheit, \& Wallace, 1991; Farah, O'Reilly, \& Vecera, 1993; Wallace \& Farah, 1992) studies help illustrate the proposed relationship between consciousness and the graded quality of representations. Farah et al. (1991) studied patients with extinction, a disorder of spatial attention, who were presented pairs of stimuli (e.g., pictures of an apple and a comb), one of which was partially occluded, and were later asked to judge whether they matched or differed. Farah et al. (1991) found that patients were not consciously aware of the degraded portion of the display and so were unable to correctly identify the stimulus but made accurate judgments as to whether the pairs of items were the same or different.

Farah et al.'s (1991) findings are consistent with prior studies (e.g., Karnath, 1988; Volpe, LeDoux, \& Gazzaniga, 1979) that provide evidence for dissociations between judgment and conscious identification. However, Farah et al. (1991) repeated the task with forced-choice questions (e.g., “did you see an apple or a comb?") and found that correct identification of the stimuli was a reliable index of the accuracy of judgments. Farah et al. (1991) concluded that identification and judgment tasks made differential demands on the kind of representation held. Thus, the findings demonstrate a correlation between the graded quality of the perceptual representation (i.e., the partially occluded stimuli) and the contents of conscious knowledge (i.e., identification of stimuli).

In order to draw the different proposals of the DGC framework together, the following discussion charts the progress of representations along the continuum as they gain strength, stability, and distinctiveness, and outlines with illustrations what form of learning occurs.

Consciousness does not have a functional role in implicit learning, and by definition there is no awareness or control over the representations formed. For example, in Roediger and McDermott's (1995) memory illusion study participants were presented with lists of words (e.g., bed, rest, awake, tired, dream, wake, snooze, blanket), all of which were associates of a target (e.g., sleep) that was itself absent from the list. In a recall test, participants falsely recollected critical nonpresented words (e.g., sleep). Participants were also given recognition tests that included three types of items: words previously shown, critical items (e.g., sleep), and noncritical items (e.g., spider) that were not presented. Each word was classified according to whether it was new or old. There was a further classification for old items, according to whether participants "remembered" or "just knew" that the item had been presented before. Roediger and McDermott found that false recognition was high for critical words (e.g., sleep) and actual studied items, whereas noncritical items were correctly identified as not having been presented. In this example, the representation of the word sleep has been activated incidentally and without any conscious awareness. Moreover, participants were unable to control this representation by preventing its inclusion in the list of remembered items and for which there was overconfidence in its acquisition.

During explicit learning, consciousness has an active and deliberate role. The representations themselves are stable, strong, and distinctive enough to be translated into declarative statements. There is conscious control of the representations because one has metaknowledge of them and of their relevance at the time of processing. A simple example of this is trying to memorize a telephone number. This process requires conscious control of the representations (i.e., the list of numbers) so that they can be accurately stored in memory and reliably recalled in the future.

In the case of automatic learning, representations have accumulated such strength that their influence on behavior is no longer controlled. In contrast to implicit learning, during automatic learning the individual is able to show metaknowledge of representations and their influence on behavior. The representations have become highly specialized because they are now optimally suited to a particular environment. For example, in the Stroop task (Stroop, 1935) participants are instructed to name the color of words presented to them, but there is interference from the automatic tendency to read the actual words, which results in slower reading times. According to the DGC framework, automatic learning is defined by an individual's ability to consciously access behavior but not the ability to control it, and as the Stroop task shows, individuals are aware of the written words but have difficulty restraining their automatic tendency to read them.

\section{Applications to Reasoning}

In this article, the DGC framework is used as an evaluative tool to contrast dual processes with a single-system account. Essentially, it describes how different forms of reasoning might occur and what the underlying relationship between these different forms is. Recasting Cleeremans and Jiménez's (2002) DGC framework as an account of reasoning, the basic tenets remain the same. Reasoning involves generating and utilizing representations to infer the validity of the claims of a proposition, decision, or judgment. As with learning, representations increase in quality along a continuum that leads to an equivalent progression in the type of reasoning, from implicit to explicit, to automatic, for which consciousness has a different functional role. The DGC framework adopts a number of proposals made by J. R. Anderson's (1993) ACT-R model, which describes some basic operations of learning and reasoning, in order to specify more clearly how the DGC framework applies to reasoning.

Anderson (J. R. Anderson \& Betz, 2001; J. R. Anderson \& Douglass, 2001; J. R. Anderson \& Sheu, 1995) views learning, categorization, problem solving, and causal inference as operations composed of production rules (these are if-then or condition-action pairs), and by extension, 
reasoning. The "if" specifies the circumstance under which the rule applies, and the "then" specifies to what, although these are not necessarily formally stated. In this sense, productions are functional units that associate the properties of a given domain (e.g., when there is $\mathrm{P}$ ) with cognitive actions (e.g., then infer Q).

Complex forms of reasoning are distinguished from simpler forms by the chains of production rules that are set to achieve particular goals; similarly, novice and expert reasoners are identified according to the complexity of the chains of production rules they use. The advantage of using ACT-R to describe reasoning within the DGC framework is that it assumes that the same procedures operate in both learning and problem solving, for which there is empirical support (Bourne, 1970; Burns \& Vollmeyer, 2002; Dienes \& Perner, 2003; Geddes \& Stevenson, 1997; Klayman \& Ha, 1987). In addition, ACT-R does not attest to functional differences between forms of skill-based automatic processing, implicit processing, and explicit processing. Finally, with more use, production rules gather strength demonstrated in the speed and reliability of performance with practice, which maps onto the assumptions that the DGC framework makes of the quality of representations.

It is important at this stage to acknowledge some of the limitations of the DGC framework. As discussed already, it describes how implicit and explicit cognitive processing occurs. Dual-process theories also make claims concerning these forms of processing, but they are particularly concerned with describing distinctions between pragmatic and formal types of inference and the evolutionary origins of reasoning, about which the DGC cannot make specific claims. Finally, the evidence from reasoning studies discussed in the remainder of this section can only provide indirect support for the DGC framework, because applying the framework to reasoning is an innovation of this article.

\section{Differences Between Dual Theories of Reasoning and the DGC Framework}

What are the differences between the DGC framework and dual-process theorists' claims? The DGC framework proposes that differences in representation generate variation in forms of reasoning without assuming a multiplesystem framework. The framework also makes an important distinction between implicit and automatic reasoning; by contrast, dual-process theorists use the terms interchangeably and take them to refer to the same process. In their definition of implicit processes, Evans and Over (1996) suggest that they "operate in parallel, are computationally extremely powerful, and present only their end products to consciousness" (p. 10). Similarly, Sloman (1996) proposes that for automatic processing "a response is produced solely by the associative system [1], a person is conscious only of the result of the computation, not the process" (p. 6). Both definitions suggest that the knowledge utilized by this system is not conscious. However, Over and Evans (1997) and Stanovich and West (2000) propose that System 1 also utilizes a type of knowledge that is acquired consciously, which through practice becomes automatic.

On the one hand, these definitions of implicit processing refer to knowledge that is acquired and utilized without awareness, but on the other hand they include knowledge that has been first learned explicitly and is then through practice applied to situations without awareness. In the learning field, these are treated as different; Berry and Dienes (1993) claim that the former is what defines implicit learning, while the latter is automatic processing or skill learning. The following discussion highlights the differences between how the DGC framework treats implicit, explicit, and especially automatic processing and that of dual-process theories by defining with examples each of the three forms of reasoning outlined.

The differences between implicit and automatic reasoning are made more apparent in the DGC framework and clarified in the following definitions. Implicit reasoning involves making a set of abstractions or inferences without concomitant awareness of them. The abstractions or inferences occur unintentionally, are not susceptible to conscious control, and are therefore not directly accessible to manipulation but are still capable of influencing explicit processes. To illustrate, Siegler and Stern (1998) presented second-graders (8-9-year-olds) with a series of arithmetic problems over eight different learning sessions. Throughout the study, all participants used a combination of strategies acquired incidentally and deliberately. Siegler and Stern used a number of measures to examine the differences between these strategies, including accuracy of performance, solution times, and verbal reports. They found no differences between implicit and explicit strategies based on accuracy of performance. Furthermore, there were no differences in working memory demands, which were measured by recording response latencies. The verbal descriptions of implicit strategies were generally poor and less detailed, and participants were unable to recognize when they had used them. However, through repeated encounters with problems on which the strategy was applicable, the representations formed became more coherent and distinct, which led to greater awareness and more accurate understanding of the strategy. In turn, participants had more control over them when they used this type of strategy.

The DGC defines explicit reasoning as having awareness of the abstractions or inferences that are made, which can be expressed as declarative knowledge. The abstractions or inferences are available to conscious control, and this allows them to be modified directly because they are accessible. There is conscious control of the representations because one has metaknowledge of them and of their relevance at the time of processing. They have a high rate of activation and can be reliably recalled from memory because they are stable enough to become registered in working memory.

Automatic reasoning is skill based and deliberately acquired through frequent and consistent activation of 
relevant information that becomes highly familiarized. This type of reasoning enables abstractions or inferences to be made without any control because the representations are enduring, well defined, and stable through repeated use. The individuals possess metaknowledge of these representations' influence and relevance to a task (J. R. Anderson, 1993), but not the opportunity to control them. For instance, when interpreting the meaning of a sentence, skilled readers process the individual words automatically (e.g., Jacoby, Levy, \& Steinbach, 1992). The processing is entirely relevant to the task and necessarily entails the recognition of their words and their meaning, but this is not invoked deliberately. When readers' recall and evaluation of the sentences was examined, they expressed accurate metaknowledge of the words they had processed automatically.

Analogous to this, a skilled reasoner or logician will automatically recognize which premises are relevant, what the correct set of inferences are that follow, and what conclusion should be drawn. This chain of inferences will, if the task is highly familiar, be executed automatically, and with some effort, the individual will be able to retrace the chain of inferences to explain how the conclusion was reached. For example, Galotti et al. (1986) compared poor, good, and expert syllogistic reasoning and found that experts responded faster than both of the other groups and demonstrated accurate metaknowledge of their reasoning behavior. Implicit reasoning is likely to result from situations where reasoners are unfamiliar with the task environment. In this case, they will recognize few features of the task as relevant and will not know why they are important. Furthermore, the chains of inferences that will lead to a conclusion are not retraceable because they are new and unfamiliar, and so no evidence of metaknowledge will be found.

The DGC framework and most dual-process theories agree that the knowledge utilized during automatic reasoning is initially acquired explicitly and becomes automatized through practice. Similarly, both types of account propose that implicit reasoning only reveals the end products of its operations but not the processes that lead to them. Crucially, the DGC differentiates between implicit and automatic forms of skill-based reasoning and provides a framework for understanding the underlying relationship between them on the basis of quality of representation.

Investigations of skill-based reasoning show that it involves both highly complex analytical processing (e.g., Galotti et al., 1986; Reingold, Charness, Pomplun, \& Stampe, 2001; Reingold, Charness, Schultetus, \& Stampe, 2001) and simple algorithms that compute frequencies (e.g., Brase, Cosmides, \& Tooby, 1998), although the evidence for this is quite controversial. The case for a highly specialized frequency algorithm (Brase et al., 1998; Cosmides \& Tooby, 1996) is based on evidence that cognitive illusions diminish when problems are framed according to a natural frequency format (Gigerenzer \& Hoffrage, 1995). Sloman, Over, Slovak, and Stibel's
(2003) alternative explanation for these beneficiary effects is in terms of the nested-set hypothesis. They assert that individuals can either construe class or category structures according to their instances, or by their internal properties. Typically, individuals make probability estimates on the basis of the properties of a category that can produce inaccurate judgments, whereas frequency formats disambiguate problems by inducing a representation of category instances and highlight how relevant instances correspond to each other. Essentially, clarifying set relations in probabilistic problems facilitates performance, and frequency formats do this, but problems where set relations are transparent without using a frequency frame are equally potent.

Dual theorists (Evans \& Over, 1996; Stanovich \& West, 2000) propose that implicit/automatic processing of System 1 is heuristic based, whereas in System 2 explicit processing is analytic. It is less clear how the findings discussed here would be accommodated within a dualtheory account, since on the one hand they are consistent with System 1 processing, in that they are examples of implicit reasoning, but on the other hand some of the findings are examples of System 2 because they demonstrate analytic processing. One view of these studies is that they show that skill-based reasoning develops in a range of domains that require high-level deductive reasoning processes, as well as simple problem-solving skills, both of which the DGC views as automatic. The DGC framework does not assign a different functional role to these types of skill-based reasoning, since in both cases the reasoning depends on the formation of higher quality representations and the degree of control that individuals have over them without reference to distinct reasoning systems.

\section{What Are the Criteria for Demonstrating Distinct Reasoning Systems?}

This section begins by describing the type of evidence that has been used to support dual-process theories of reasoning and discusses, in some detail, tasks that have provided this sort of evidence. It also includes a brief analysis of the implications of the findings for dualprocess theories; however, the main evaluation is reserved for the final section of this article. The key evidence is summarized, and the claims made by dual-process theories, as well as the proposals offered by the DGC framework, are examined in relation to it.

Support for dual-process theories comes from a wide range of studies (e.g., categorization, judgment and decision making, problem solving, probabilistic, inductive, and deductive reasoning). Given such a range, this article focuses on evidence from three particular tasks (Wason's selection task, the conjunction problem, and syllogisms) taken from different domains of reasoning research. These tasks have been chosen because they have been widely researched and generate robust findings that the current dual-process theories have found to be highly supportive of their claims. In addition, these tasks are representa- 
tive of different forms of reasoning: Conditional reasoning, decision making, and deductive reasoning further demonstrate the generality of the dual-process account. The findings from the studies described here are classed under four criteria (Criterion S, individual differences, implicit vs. explicit processing, and neuroanatomical differences) that have been used to identify the two systems.

Criterion S. Sloman's (1996) Criterion S, discussed earlier, outlines types of situations that demonstrate that the two reasoning systems operate simultaneously but lead to diverging outcomes. Sloman (1996) specifies that Criterion $\mathrm{S}$ is also fulfilled when an individual's initial response still exerts some influence although a second answer provided either by them or the experimenter is known to be more acceptable.

Individual differences. In addition to Sloman's (1996) Criterion S, Stanovich (1999) predicts that "large differences in cognitive ability will be found only on problems that strongly engage both reasoning Systems and in which the reasoning Systems cue opposite responses" (p. 143). An important aspect of such a task is that differences in cognitive ability map onto the two types of responses. Those providing a normatively correct response also show higher cognitive ability compared with individuals whose answer is intuitively based.

Implicit versus explicit processing. Dissociations between implicit and explicit processing have been investigated in studies that have used protocol measures, transfer tasks, and tutoring. These measures indicate that, unlike explicit processing, implicit processing is difficult to access, and so participants are unable to express and control when and where it is applied. In addition, failure to improve performance following tutoring supports the view that participants are unaware of the processes that contribute to their responses and so are unable to address them. Measuring the effects of tutoring in transfer tests also reveals that knowledge applied implicitly is utilized in domains specific to where it was first acquired.

Neuroanatomical differences. Clinical and neuroimaging studies suggest that underlying neuroanatomical differences between the two forms of reasoning are mediated by different hemispheric regions of the brain.

\section{Wason's Selection Task}

In Wason's (1968) first published study of what now is referred to as the selection task, participants were asked to decide which of the four cards (A [P], K $[\neg \mathrm{P}]$, $4[\mathrm{Q}]$, and $7[\neg \mathrm{Q}]$ ) should be turned over to prove whether the conditional statement "if there is a vowel on one side (antecedent), then there is an even number on the other (consequent)" is true or false. The correct solution requires the selection of $A(P)$ and $7(\neg Q)$, since they are the only combination that can potentially refute the statement. However, most participants tend to select the A (P) and $4(\mathrm{Q})$ cards, which have been found to be a highly robust choice. The first theory to account for this tendency claimed that individuals demonstrated a bias toward con- firming the statement (Wason, 1969), which was later shown to be a bias for matching named cards with example cards (Evans, 1972). The "matching" phenomenon has since been widely researched (for a review, see Evans, 1998); however, as yet there is little agreement as to what underlying process generates this response.

Thematic or "contextualized" versions of reasoning tasks are examples where the task is embedded in a context that makes references to real-world situations, and these were devised to facilitate performance (e.g., Wason \& Shapiro, 1971). Often, contextualized versions include deontic statements (e.g., "if you tidy your room, you can go out to play"), and these reliably generate a high proportion of correct responses (e.g., Cheng \& Holyoak, 1985; Griggs \& Cox, 1982; Sperber, Cara, \& Girotto, 1996).

The general view (e.g., Manktelow \& Over, 1995) is that contextualized versions make references to situations relevant to the reasoner. In such framings of the task, reasoners are aware of exceptions and so are able to select cards that undermine the statement. In this sense, deontic and indicative versions are not comparable tasks because they pose different questions: In indicative versions, participants are asked to falsify the rule, whereas in deontic versions they are told to look for violations.

\section{Criterion S}

While there are no actual empirical tests of this criterion, Sloman proposes that evidence from studies of indicative selection tasks satisfies Criterion S. Through System 1, a computation of similarity between cards named in the rule and example cards generates the initial response of PQ selections. Corrective methods such as tutoring encourage System 2 rule-based processing, but the initial matching response is highly salient and still influences participants' responses to the task. Early tutoring studies provide strong support for Sloman's proposals. Wason (1968) first introduced tutoring procedures or "therapies" to invoke insight into the task. In general, early studies using tutoring techniques failed to improve performance (e.g., Johnson-Laird \& Wason, 1970; Wason \& Shapiro, 1971). When performance did significantly improve, following tutoring, it did not transfer to other framings of the task (e.g., Wason, 1969; Wason $\&$ Golding, 1974), thus, further suggesting that the knowledge is context specific and therefore mediated by associative processing consistent with a dual-process account.

An alternative explanation for these results is that participants were unresponsive to early tutoring methods because the techniques were inadequate, and they simply failed to identify and address problems related to correctly representing and understanding the task. In abstract versions of the task, the vast majority of participants $(35 \%-60 \%)$ selected matched cards. However, a sizable proportion selected the $\mathrm{P}$ card $(20 \%-35 \%)$, and a smaller proportion chose a range of other card combinations $(10 \%-20 \%)$. Rationalist accounts claim that the different card selections reflect differences in partici- 
pants' interpretations of the conditional statement but from these they are reasoning logically (Fillenbaum, 1976; Osman \& Laming, 2001; Stenning \& van Lambalgen, 1999).

Recent tutoring studies that tailor their techniques to the individual's construal of the statement have found improvements in performance that also transfer to complex propositional tasks (e.g., Margolis, 2000; Osman, 2002; Stenning \& van Lambalgen, 2001). For example, Stenning and van Lambalgen (2001) identified a variety of interpretations (e.g., "one side-other side" was taken to mean "back and front") of the conditional statement and the relevancy of the different cards, which were later addressed in tutoring sessions. They found that correct performance transferred to other abstract versions of the task, as well as to a complex conditional problem. This task involved four example cards, and participants were asked to choose from them in order to falsify two conditional statements simultaneously. Osman (2002) used similar techniques to Stenning and van Lambalgen (2001) and achieved transfer to a deadline version of the abstract task (responses under limited time conditions) and to one that required participants to generate and test their own version of the selection task using statements and examples they devised themselves.

Criterion S proposes that the shifts from matched to correct card choices found in studies of tutoring result from a switch from System 1 to System 2 reasoning. It also provides an account of why tutoring fails. However, it is unclear whether Criterion $\mathrm{S}$ treats other erroneous card choices (e.g., P, $\neg$ PQ) as products of an associative system, and by the same token whether shifts from these to correct responses are indicative of changes from associative to rule-based reasoning. Techniques used in tutoring studies vary; however, what is common to recent studies of tutoring is that the large improvements in performance are the result of clarifying the task requirements and removing the ambiguity in the conditional statement (e.g., Cheng, Holyoak, Nisbett, \& Oliver, 1986; Klaczynski, Gelfand, \& Reese, 1989; Stenning \& van Lambalgen, 2001). One advantage of the rationalist account is that it accommodates a wide range of card-selecting behavior by claiming that different choices are evidence of different construals of the statement. The underlying reasoning system does not change when individuals improve in their performanceit is the interpretation of the task that changes.

\section{Individual Differences}

There is good evidence that cognitive ability and performance on the abstract selection task correlate (e.g., Klaczynski, 2001; Stanovich \& West, 1998b). This supports Stanovich and West's (2000) claims that the two reasoning systems often cue different responses that can be discriminated according to high- and low-cognitive ability scores. Stanovich and West (2000) also claim that deontic versions do not correlate with SATs, because both System 1 and System 2 cue the correct response, so participants of different cognitive abilities will perform optimally.
Newstead, Handley, Harley, Wright, and Farrelly's (2004) study aimed to replicate Stanovich and West's (1998b) findings. They found that the rational-experiential inventory (REI) designed by Pacini and Epstein (1999), which Newstead et al. (2004) used to measure intuition and rational thinking, failed to correlate with performance on the selection task. Measures of intelligence (AH5 intelligence test) did correlate with responses to the task; however, this was not based on performance, but on the consistency of the cards selected across different versions, irrespective of what that combination was. High cognitive ability correlated with selections of the $(\mathrm{P})$ card in abstract versions, a finding also reported by Stanovich and West (1998b). Conventionally, the selection of the $\mathrm{P}$ card has been taken to indicate poor performance, whereas a card choice that includes the combination $\mathrm{P}, \mathrm{Q}$, and $\neg \mathrm{Q}$ is suggestive of partial insight (Wason \& Johnson-Laird, 1970), which Stanovich and West (1998b) found correlated with low cognitive ability. Newstead et al.'s (2004) findings suggest that cognitive ability is predictive of certain types of response pattern but that they do not clearly map onto the kinds of differences that Stanovich and West (2000) take as support for different reasoning systems.

\section{Implicit Versus Explicit Processing}

Protocols. In evaluating the evidence from protocol measures, two related issues need to be considered. First, do the accounts that participants generate accurately correspond with behavioral evidence of their mental processes? Evans (Evans \& Wason, 1976; Wason \& Evans, 1975 ) found that the verbal protocols that participants gave of their card selections were incompatible with their actual card choices, and were post hoc rationalizations rather than accurate descriptions of their reasoning behavior. Moreover, there was no difference between participants' confidence in the reasons given for valid and for invalid card choices, suggesting that confidence ratings of rationalizations were independent of the cards selected because participants do not have access to the processes that govern their own selections.

Later studies have reported associations between protocols and reasoning behavior. For example, Berry (1983) used protocol techniques to investigate cross transfer of correct responding in abstract and contextualized versions. She found that justifications for card selections during concurrent protocols made participants aware of the correct attributes of their own reasoning process, and produced transfer from contextual to abstract and abstract to contextual versions of the task. Despite unsuccessful attempts to replicate Berry's (1983) findings concerning cross transfer (Chrostowski \& Griggs, 1985; Klaczynski et al., 1989), which has been thought to result from differences in the type of contextual material used, in Hoch and Tschirgi's (1983) study a correspondence was found between participants' written protocols and their card choices in both abstract and contextual versions. In a later replication, Hoch and Tschirgi (1985) compared card choices before and after written protocols 
between students at different levels (high school, bachelor's and master's degree) of education. Bachelor's and master's students were more likely to improve in performance after they explained their selections. Hoch and Tschirgi (1985) suggested that educated participants had more accurate representations of the problem structure to begin with and so, through protocols, were better at identifying the errors in their thinking.

The second issue related to using protocol measures is whether they should be treated as data in their own right. There are mixed views on the credibility of data gathered from protocols: Nisbett and Wilson (1977) consider them to be an unreliable source of support for implicit processes, but Ericsson and Simon (1980) suggest that protocols are a valid way of demonstrating dissociations between implicit and explicit processes. Certainly, early protocol studies provide persuasive evidence for dual-reasoning systems and motivated the development of one of the earliest dual-process theories of reasoning (Wason \& Evans, 1975). More recent studies (Green \& Larking, 1995; Platt \& Griggs, 1995) that have used protocol measures indicate that they are more usefully employed when supplementing other measures of reasoning, particularly when developing ways to facilitate performance. Overall, the findings from work using this type of measure suggest that participants do not spontaneously offer informed knowledge of the processes by which they arrive at their answers but with some cuing are able to articulate and capitalize on this knowledge.

Inspection times. Dissociations between implicit and explicit processes have also been studied using response time measures. Evans, Ball, and Brooks (1987) were the first to develop a measure of attentional biases, which are thought to influence card choices (Evans, 1984). They recorded the order in which decisions for card selections and rejections were made and found that participants first decided on cards they would select, followed by those they would reject. This suggested that preconscious biases direct attention toward cards deemed relevant through linguistic cues from the task; if, instead, the task was processed analytically, all four cards would be considered equally. In a later study by Evans (1996), participants were presented all four cards onscreen and recorded the overall time taken for the inspection and selection (measured by the time taken to move a mouse pointer to a card) of each individual card. Evans found that across abstract, negative, and thematic versions, participants spent more time inspecting cards they selected than those they rejected, a finding replicated by Ball, Lucas, Miles, and Gale (2003) and Roberts and Newton (2001). Evans (1996) claimed that card selections are the result of heuristic processes that are invoked automatically, whereas analytic processes that require time are invoked later and are recruited to rationalize the decisions made.

Roberts and Newton (2001) proposed that with the appropriate methodology, inspection time effects are highly replicable, but that with modifications (e.g., blacking out the cards until a card became highlighted only in the event of moving the mouse pointer across it, or including yes-no decision buttons under each card, or a combination of both) the effects are muted or in some cases reversed (Roberts, 1998). Roberts and Newton conclude that their evidence supports Evans's (1989) heuristic-analytic account but is also consistent with other single-process accounts such as mental models theory. Johnson-Laird and Byrne's (1991) mental models theory proposes that the initial construction of representations is often incomplete, but with time or the appropriate context they become "fleshed out," leading to correct responses. Thus, it predicts that increasing the time to think about the task encourages participants to expand the number of representations, which alternatively describes the changes from heuristic to analytic processes.

\section{Neuroanatomical Differences in the Selection Task}

Golding's (1981) study provided the earliest evidence for the role of the left hemisphere (LH) in contentindependent reasoning. Golding examined cards chosen in an abstract version of the selection task by patients with right hemispheric (RH) and LH damage. Half the patients with RH damage responded correctly to the abstract task, compared with only one patient with LH damage. Golding claimed that patients with RH damage, including impairment to visual processing, were prevented from making a perceptual match between the statement and the example cards and instead relied on verbal reasoning ability.

Houde et al.'s (2001) positron emission tomography (PET) study compared two groups of normal participants, one receiving a short logic tutorial, and the other a "logicoemotional" training session designed to elicit feelings in conjunction with potential errors that could be made. The groups were scanned while performing abstract versions of the selection task before and after training. Houde et al. found that participants were more receptive to logicoemotional training compared with pure logical training, as indicated by a higher proportion of correct selections. In addition, they found that the right ventromedial prefrontal cortex was only activated in the logicoemotional group, which has been associated with decision making, emotion, and feeling (S. W. Anderson, Bechara, Damasio, Tranel, \& Damasio, 1999). In contrast to Golding's (1981) claims, Houde et al. proposed that deductive reasoning depends on $\mathrm{RH}$ regions that are also devoted to emotion. Knauff, Mulack, Kassubek, Salih, \& Greenlee's (2002) fMRI study of spatial brain regions in deductive reasoning used relational and conditional problems. For both types of problem, activation of RH regions was associated with the formation of spatial representations, and occipito-parietal pathways were thought to support mental imagery, which is, again, inconsistent with Golding's claims.

Neuropsychological studies using conditional reasoning tasks provide conflicting results as to which hemispheric regions are involved in particular forms of reason- 
ing. This may in part be the result of the variability in the materials and the techniques used. However, the Houde et al. (2001) and Knauff et al. (2002) studies vary significantly in the actual task administered but agree on the brain regions involved in abstract conditional reasoning.

\section{Conjunction Problem}

Tverksy and Kahneman (1983) presented participants with a description of "Linda" from which the following is an abbreviated version: "As a student, she was deeply concerned with issues of discrimination and social justice, and participated in anti-nuclear demonstrations" (p. 297). The participants were then asked to rank eight statements (e.g., "Linda is active in the feminist movement," "Linda is a bank teller," "Linda is a bank teller and is active in the feminist movement") according to how probable they were. The correct judgment relies on participants applying the conjunction rule, which states that the probability of the conjunction cannot exceed either of its constituents. Tverksy and Kahneman (1983) found that $80 \%$ of participants committed the conjunction fallacy by rating "bank teller and feminist" as more probable than either "bank teller" or "feminist" alone.

Tverksy and Kahneman (1983) claimed that the fallacy was the result of an intuitive strategy, termed the representativeness heuristic, which participants used to assess "the degree of correspondence between a sample and a population, an instance and a category, an act and an actor or more generally an outcome and a model" (p. 295). This problem has consistently demonstrated reasoners' errors in judgment and, like the selection task, has generated a host of research, much of which has been directed toward understanding why the fallacy is committed.

\section{Criterion S}

Sloman (1996) proposed that despite knowledge of the conjunction rule, participants still tend to commit the fallacy, and this satisfies Criterion $\mathrm{S}$ because it is indicative of the two reasoning systems cuing contradictory responses.

For example, Tversky and Kahneman $(1974,1983)$ examined whether prior knowledge of the rule would lead to improvements in performance. They presented the task to three groups: naive (undergraduates from Stanford University), informed (graduate students in psychology), and sophisticated (doctoral students in decision science program) with different experiences of probabilistic rules. In all three groups, $85 \%-89 \%$ violated the conjunction rule.

As with the selection task, individuals are generally insensitive to corrective measures. This suggests that what generates the fallacy is difficult to alter, because of its associative basis, which may be why participants continue to lapse back into their initial beliefs after being informed of their erroneous thinking (e.g., Epstein, Donovan, \& Denes-Raj, 1999).

Studies that have attempted to induce insight into the task through training procedures propose that the fallacy arises from misrepresenting the problem (e.g., Dulany \&
Hilton, 1991; Hilton, 1995; Margolis, 1987; Markus \& Zajonc, 1985). Morier and Borgida (1984) examined the extent to which they could reduce the fallacy by making the instructions more explicit. Participants were asked to give probability estimates for each statement instead of ranking them, and to give reasons for their estimates. Often, respondents claimed that they used an averaging rule that weighted the traits of the character described. Morier and Borgida found that from this, they could attenuate the degree to which the fallacy was committed by tailoring instructions to avoid application of the averaging rule, which they found had a marked effect on performance but did not completely eliminate the fallacy. They concluded that participants' misunderstandings of the instructions influence the extent to which the fallacy is committed.

Agnoli and Krantz (1989) found that performance increased following a short tutorial on Venn diagrams that was designed to encourage thinking about category extensions rather than the category features of Linda's character. And Fong (Fong, Krantz, \& Nisbett, 1986; Fong \& Nisbett, 1991) reported the effectiveness of statistical training on facilitation of correct performance on probabilistic tasks. Fong and Nisbett found that participants possess rudimentary abstract knowledge of statistical rules such as the law of large numbers and conjunction, and that because they possess some statistical intuitions, formal training procedures are able to build directly on such knowledge. This is consistent with studies that include protocol measures (e.g., Morier \& Borgida, 1984; Stolarz-Fantino, Fantino, \& Kulik, 1996), which show that as well as reporting the use of similarity as a strategy, participants develop strategies that utilize superficial knowledge of probabilistic rules to solve the task.

There is evidence that linguistic factors contribute to generating the fallacy, and that correcting misunderstandings can improve performance. However, other factors apart from ambiguity of the task framing generate the fallacy. Sides, Osherson, Bonini, and Viale (2002) attempted to reduce the source of misinterpretation by presenting unambiguous conjunctive statements in a betting paradigm in which participants bet on the conjunctive or one of its conjuncts. They found that the fallacy was committed as frequently in the betting paradigm as in the standard version. This provides compelling evidence for Criterion $\mathrm{S}$, which suggests that the fallacy is generated by a highly resistant bias resulting from associative processing.

\section{Individual Differences}

Stanovich and West (1998a) proposed that the Linda problem presents a host of features that support pragmatic inferences (e.g., Adler, 1991; Dulany \& Hilton, 1991) invoked by System 1. Participants who solve the task correctly overcome the more obvious aspects of the task and apply the conjunction rule by using System 2 processing. In their study, Stanovich and West (1998a) compared performance and SAT scores in a standard version of the Linda problem and two transparent ver- 
sions (i.e., Reeves \& Lockhart's [1993] job problem and Fiedler's [1988] student problem). Unlike in the selection task, in which they found that performance on simpler deontic versions was uncorrelated with ability, because both systems cue the same response, Stanovich and West (1998a) found that performance on the job problem correlated with ability. These findings can be taken to show that easier versions of the Linda problem may actually engage System 2 processes, and this is why performance correlates with cognitive ability. However, they are also consistent with Yates and Carlson's (1986) findings, showing that individuals use a variety of informal statistical rules that often lead to an incorrect response but can also solve the task correctly, which does not require a two-system explanation.

\section{Implicit Versus Explicit Processing}

Studies that use protocol measures extend much of the findings from tutoring studies suggesting that participants invoke a variety of misrepresentations of the problem. Morier and Borgida (1984) categorized written protocols according to two categories and found that most participants explained their responses according to typicality ("she sounds like a feminist, which makes it more likely than just bank teller"), and the remainder by way of an averaging rule ("I averaged the two answers"). Yates and Carlson's (1986) protocol study uncovered participants' different understandings, from which a variety of strategies were developed to solve the problem. They identified seven types of strategies, some of which they proposed were "formaliztic": judgments made according to a combination of probabilistic rules, such as averaging. These findings suggest that protocols are an accurate way of determining the methods used to solve the problem, particularly because the types of misinterpretations identified in these studies have been corrected, leading to improved performance (e.g., Fisk \& Pidgeon, 1996). This implies a correspondence between Systems 1 and 2. Furthermore, Yates and Carlson's findings, along with tutoring studies (Benassi \& Knoth, 1993; Betsch \& Fiedler, 1999; Crandall \& Greenfield, 1986; Morier \& Borgida, 1984) suggest that improvements in performance can occur when participants supplement one type of rule-based reasoning (e.g., applying an averaging rule) for another (e.g., the conjunction rule). This contrasts the dual-process account, which would claim that in order to induce correct responses the rule-based system is required to override the heuristic-based system.

Both protocol and tutoring studies suggest that participants are aware of their reasoning processes, even in cases where it is evident that they are using a type of heuristic. Moreover, in tutoring studies (Benassi \& Knoth, 1993; Crandall \& Greenfield, 1986), often where there is no direct mapping from tutoring session to test items, participants still are able to transfer newly acquired knowledge to similar and disparate task domains. Again, this indicates that participants can access and change what seems to be a highly resistant bias.

\section{Neuroanatomical Differences in Judgment and Decision Making}

Studies of schizophrenic patients' reasoning (e.g., Kemp, Chua, McKenna, \& David, 1997) that, among many tasks, included the Linda problem, found that performance was comparable with that of normals. This indicates that neurological insult does not necessarily affect judgments made in typical probabilistic tasks and that the rudimentary processes involved are spared, which is consistent with some dual-reasoning theorists' claims. However, other studies (Dudley, John, Young, \& Over, 1997; Huq, Garety, \& Hemsley, 1988) have found that neurological deficits do change the nature of the reasoning process involved in judgment-based tasks (e.g., judging the outcome of coin tosses). Dudley et al. (1997) found that, while their general reasoning ability was not impaired, schizophrenic patients required less information when making a statistical judgment. This was interpreted as resulting from the patients' pathology, which made them mistrustful of the information presented, and increased reliance on their own experience to form judgments. As with the selection task, discrepancies in reported findings might be due to differences in the type of material used and the population of patients tested.

\section{Syllogism}

Syllogisms are complex arguments that include two statements (premises) and a conclusion: For example, all artists are beekeepers, all beekeepers are chemists, therefore all artists are chemists. Syllogisms vary in type and complexity, and there is a substantial corpus of studies investigating the types of deductive reasoning that are involved in solving them (for a review, see Johnson-Laird \& Byrne, 1991).

\section{Criterion S}

One of the most robust effects in syllogistic reasoning is belief bias. This is the tendency to base an assessment of the validity of a syllogism on the believability of its content. When belief and logic agree, bias facilitates the logical response. For example, Evans et al. (1983) found that most participants correctly assessed as invalid the argument "No police dogs are vicious, some highly trained dogs are vicious, therefore some police dogs are not highly trained." When belief and logic disagree, participants often draw erroneous conclusions. Evans et al. (1983) found that the majority of participants incorrectly accepted as valid the argument "No addictive things are inexpensive, some cigarettes are inexpensive, therefore some addictive things are not cigarettes." Sloman (1996) proposed that the latter effect satisfies Criterion S because conflicting responses generated by the different reasoning systems cue contradictory conclusions as to the syllogism's validity.

Accounts of bias differ in terms of where in the reasoning process it is generated. This, in turn, has implications as to whether the bias is consistent with the conditions of Criterion S. Given the primacy of System 1, evidence of 
the bias occurring early in reasoning supports the proposal that it results from automatic associative processes (consistent with Criterion S), whereas evidence suggesting the bias comes late in reasoning (suggestive of slower System 2 processes) would trace the bias to analytic processes and therefore would be inconsistent with Criterion $\mathrm{S}$.

Evans (Evans et al., 1983; Evans \& Pollard, 1990) found that participants tended to check the conclusion of a syllogism, and according to its believability, would accept it without further evaluation. Only when it mismatched their own beliefs would they scrutinize the conclusion. These findings were explained in terms of the selective scrutiny two-stage theory (Evans, 1989; Evans \& Pollard, 1990) and satisfy Criterion S. The theory proposes that the bias occurs early in the encoding stage and focuses reasoners' attention on the conclusion rather than on the premises, thus preceding and preempting analytical reasoning.

Johnson-Laird and Byrne's (1991) mental models theory claims that solving syllogisms involves the generation of models (mental analogues of the premises) from which conclusions are generated and evaluated according to their consistency with the models. Johnson-Laird and Byrne proposed that the belief bias effect is produced at the final response stage of the reasoning process, where believability influences the generation and evaluation of the conclusion (e.g., Markovits \& Nantel, 1989; Oakhill, Garnham, \& Johnson-Laird, 1990; Oakhill \& Johnson-Laird, 1985).

Newstead, Pollard, Evans, and Allen (1992) investigated the predictions of three theories: selective scrutiny, mental models, and their own misinterpretation necessity model, which predicted that the bias occurs late in the response stage. Their results strongly supported the mental models approach. They found that participants did evaluate the arguments; however, poor understanding of logical necessity, combined with logically indeterminate conclusions, led to reliance on beliefs and failure to generate alternative examples, which would have helped assess the validity of the conclusion.

Many of the findings from studies of belief bias have indicated that when solving contentful syllogisms, participants begin with some rudimentary analytical processing, and that the source of the bias is located at the evaluation stage of reasoning. This contrasts with one of the conditions set by Criterion $\mathrm{S}$, which proposes that for believable conclusions associative processes are dominant and fast, which is why they precede analytically based evaluations of the problem.

\section{Individual Differences}

In their studies of individual differences, Stanovich and West (Sá, West, \& Stanovich, 1999; Stanovich, 1999; Stanovich \& West, 1998c) found that measures of intelligence were a strong predictor of performance on contentful syllogisms. Participants making belief bias errors, which Stanovich and West claim are generated by System 1, scored lower on tests of general intelligence, whereas participants of high cognitive ability evaluated syllogisms analytically, leading to correct performance.

Other studies investigating correlations between intellectual ability and performance on syllogistic reasoning have found that the number of models and alternative conclusions generated were more reliable predictors of performance (Newstead, Thompson, \& Handley, 2002; Torrens, Thompson, \& Cramer, 1999). Newstead et al. (2002) used a range of measures (e.g., rationality, experientiality, creativity, generation of alternatives, motivation) and found that the number of alternative models falsifying a conclusion generated reliably predicted performance on syllogistic tasks, which is consistent with findings reported by Galotti et al. (1986). Newstead et al. (2002) claimed that task variables such as instructions, content, and the complexity of the syllogism affected individuals' willingness to produce alternatives. Their findings suggest that the number of alternatives generated was more reliable as an index of performance than measures of cognitive ability.

\section{Implicit Versus Explicit Processing}

Evans et al.'s (1983) study investigated whether the conflicts between heuristic and analytic processes found in Wason and Evans's (1975) study of the selection task were also present in syllogistic reasoning. They coded participants' protocols according to the different possible combinations of references that individuals made to conclusions and premises of the syllogisms they were presented. Evans et al. (1983) concluded that those showing a belief bias often rationalized their responses by referring to irrelevant information, lending support to their dual-process account.

Other studies (e.g., Bucciarelli \& Johnson-Laird, 1999; Ford, 1995) have found that participants' protocols are consistent with behavioral evidence of their mental processes, suggesting that individuals have insight into their reasoning processes. Galotti et al. (1986) found that the protocols from three groups of reasoners (poor, good, expert) provided direct evidence of differences in their performance. Participants were able to report the number of deduction rules they possessed and the type of misinterpretations of the premises they had, both of which corresponded to their level of performance. In addition, Ford found that verbal protocols, along with the diagrams that participants constructed, indicated the types of cognitive styles (i.e., visual or verbal representations of premises) that they developed to solve syllogisms.

\section{Neuroanatomical Differences in Syllogistic Reasoning}

Deglin and Kinsbourne (1996) examined neurologically impaired patients' (i.e., schizophrenics and manic depressives) performance on contentful syllogisms. Each patient was tested three times, once before ECT (electroconvulsive therapy), then once with ECT-induced RH and LH suppression. Prior to ECT, and with RH suppression, $86 \%$ responded with logically correct answers. When the 
LH was suppressed, 79\% responded correctly. Deglin and Kinsbourne concluded that the two hemispheres supported separate reasoning mechanisms. The LH was claimed to mediate formal logical operations akin to System 2 type processing, consistent with Golding's (1981) findings, whereas the RH was dominant in contextdependent reasoning and was unable to decouple beliefbased knowledge associated with System 1 processing.

Goel's (Goel, Buchel, Frith, \& Dolan, 2000; Goel \& Dolan, 2001; Goel, Gold, Kapur, \& Houle, 1997) neuroimaging studies lend further support to Deglin and Kinsbourne's (1996) claims. Normal participants were asked to assess the validity of contentful and abstract syllogisms under time pressure conditions. Goel et al. (2000) found that when participants solved contentful syllogisms, brain regions in the LH were activated (e.g., ventral networks involving temporal and frontal regions); these have been found to be associated with syntactic and semantic processing. In participants solving abstract syllogisms, Goel et al. (2000) found patterns of right parietal activity associated with spatial processing, suggesting that they had represented and manipulated spatial information.

When comparing the activation of brain regions during probabilistic and deductive reasoning, Osherson's (Osherson et al., 1998; Parsons \& Osherson, 2001) PET studies found that RH brain regions were exclusively active during deductive reasoning, and $\mathrm{LH}$ regions were uniquely activated when reasoning with probabilistic material. Parsons and Osherson propose that the large inconsistencies between their findings and those of Goel are the result of differences in the stimuli and in the instructions of the syllogistic tasks used. Participants in their study were presented with a series of contentful deductive and probabilistic syllogisms and asked to evaluate their validity. Reasoning on probabilistic syllogisms activated brain regions (e.g., left inferior formal areas, parahippocampal and medial temporal areas) associated with the recall and evaluation of a range of semantic knowledge, whereas deductive reasoning activated regions (e.g., right basal ganglia, thalamus, and frontal cortex) associated with the transformation of mental representations, working memory, executive strategy, and rule-based learning.

Studies, both clinical and imaging, suggest that each hemisphere performs functionally distinct types of reasoning, but the conflicting findings described here imply that using this evidence to adjudicate between singleand dual-process theories is still premature.

\section{Summarizing the Evidence}

This article began by discussing the distinctions that reasoning theorists have proposed. It also introduced a new framework that could alternatively account for differences in forms of reasoning and then presented evidence from tasks that have supported dual-process theorists' claims. The aim of this final section is to summarize and draw together key evidence from these tasks and discuss their relation to the main issues that distinguish dualprocess theories and the DGC framework.

Criterion S. Dual-process theorists (Sloman, 1996; Stanovich \& West, 2000) claim that certain tasks (e.g., abstract selection task, the standard Linda problem) generate conflicts that result in the two reasoning systems cuing contradictory responses. It is said that the two systems act simultaneously and that the faster of the two generates the first response, and the second system, which is slower to act, generates the correct response. Evidence from early tutoring studies of the selection task, which is claimed to satisfy Criterion $\mathrm{S}$, is alternatively explained by tutoring methods failing to take into account the different types of erroneous beliefs that participants have (e.g., Stenning \& van Lambalgen, 1999).

In the Linda problem, which also satisfies Criterion S, conflicts are thought to arise between the correct understanding and the initial highly compelling response, which still exerts its influence over participants' later understandings of the task (e.g., Epstein et al., 1999; Tversky \& Kahneman, 1983). Alternatively, the DGC framework proposes that many individuals find some rule of inference that takes the most salient premises first from which to form an answer, and with time arrive at the correct answer. This is achieved by recombining the premises to form a different or more complex set of inferences that leads to the correct answer. The first answer is still highly influential because it is simple and is based on the most prominent features of the task, which are more likely generalizable. For example, Sides et al.'s (2002) study, which provides convincing evidence for dual-process accounts of the conjunction fallacy, shows that individuals have difficulty overlooking their initial erroneous belief. Even when sources of ambiguity from the instructions were removed, the fallacy was found to generalize to wider expressions of "and" than that of its original formulation, to the extent that participants bet on the conjunctive even when there was a monetary incentive not to do so. Here, the initial beliefs have become strongly represented, which is why, when reconsidering the task, participants cannot fail to revisit their initial inferences because these are more distinctive than the more complex specialized chain of inferences required to achieve the correct solution (J. R. Anderson, 1993; Holland, Holyoak, Nisbett, \& Thagard, 1989).

Criterion $\mathrm{S}$ assumes that System 1 generates the initial erroneous belief. But there are examples of more than one type of initially formed erroneous belief, and these can arise from what can be viewed as analytical type reasoning (e.g., Bucciarelli \& Johnson-Laird, 1999; Morier \& Borgida, 1984; Stenning \& van Lambalgen, 1999; Yates \& Carlson, 1986). The advantage of the DGC framework is that it does not differentiate between types of erroneous understandings according to whether they are heuristic or analytic. The same underlying production rule can generate a variety of erroneous responses as a result of the features of the task that an individual considers relevant. Moreover, because of this, the DGC frame- 
work accommodates individuals' different initial erroneous construals that can be analytic (Stenning \& van Lambalgen, 1999; Yates \& Carlson, 1986) as well as heuristic (Morier \& Borgida, 1984), and in turn, accounts for the successes of tutoring studies that identify and correct these different construals, the benefits of which transfer to similar and different task domains (e.g., Agnoli \& Krantz, 1989; Osman, 2002; Stenning \& van Lambalgen, 2001).

Criterion S stresses that there are many examples of reasoning tasks that cause conflicts of interest between initial and later inferences. Such a view highlights the fact that individuals select out particular features of a task to test, and that in many cases particular features of a task are found to be more salient than others. This provides important grounds for categorizing different reasoning tasks. However, there is evidence suggesting that the conflicts identified may not necessarily arise from distinct reasoning systems but can instead be interpreted as a result of the varying strengths of the representations utilized while reasoning.

Individual differences. Stanovich and West (2000) predict that in tasks where the two systems cue different responses, high scores of cognitive ability suggest the use of analytic processes. By contrast, lower scores of cognitive ability suggest that individuals are reliant on heuristic processes that often lead to incorrect responses. These claims have been supported by a number of studies (e.g., Klaczynski, Gordon, \& Fauth, 1996; Stanovich $\&$ West, 1998b, 1998c). The DGC framework proposes that what differentiates these groups is the amount of production rules and in turn, their complexity, and not whether heuristic and analytic forms of reasoning are applied. Studies of deductive reasoning (e.g., Newstead et al., 2002; Torrens et al., 1999) suggest that alternative measures (e.g., the number of counterexamples to initial conclusions generated and evaluated) predict performance above and beyond measures of cognitive ability. Similarly, Galotti et al.'s (1986) findings suggest that the frequency of alternative conclusions generated in syllogistic reasoning tasks reliably indexed individuals with differing levels of reasoning expertise.

Stanovich and West (2000) also predict that System 2, identified by high scores of cognitive ability (e.g., SAT), is slow and very cognitively demanding, whereas System 1, identified by poor scores of cognitive ability, is rapid and does not drain cognitive resources (e.g., Epstein, Pacini, Denes-Raj, \& Heier, 1996; Stanovich \& West, 1998a, 1998b, 1998c). The DGC framework proposes that the different types of reasoning are dependent on the representations from which participants reason, and this is not reliably differentiated according to the demands made on the central executive. For example, the DGC proposes that skilled reasoners demonstrate a type of automatic reasoning that is dependent on well-formed representations of highly specialized rules; these have acquired strength and become attuned to the circumstances in which they apply. Comparisons between poor and skilled reasoners suggest that in any given task, some demonstrate knowledge that is highly familiarized and results in rapid responses. Galotti et al. (1986) reported that experts performed faster than poor and good reasoners. Furthermore, studies of expertise in highly complex task domains (e.g., forecasting probable odds in a betting environment) suggest that individuals utilize a type of analytical processing that is uncorrelated with measures of intelligence (e.g., Ceci \& Liker, 1986; Ericsson, Krampe, \& Tesch-Römer, 1993) and makes little demand of executive functions.

Clearly, there is evidence to suggest that measures of performance on reasoning tasks correlate with tests of cognitive ability (e.g., Guilford, 1959; Klaczynski et al., 1996; Stanovich \& West, 1998b), but they can be interpreted as relating to differences in degree rather than in the kind of reasoning system used.

Implicit versus explicit processing. One of a number of empirical routes to examining dissociations between implicit and explicit forms of reasoning has been to use protocol measures (e.g., Evans et al., 1983; Wason \& Evans, 1975). These are designed to show a lack of correspondence between verbal/written reports and behavioral data. There is much supporting evidence of dissociations of this kind in studies of the selection task (e.g., Evans \& Wason, 1976; Wason \& Evans, 1975) and syllogistic reasoning (e.g., Evans et al., 1983). However, others have shown that protocols are a good index of the type of knowledge that individuals bring to bear on a task (e.g., Galotti et al., 1986; Goodwin \& Wason, 1972; Stenning \& van Lambalgen, 1999).

One crucial difference between studies that report a correspondence and those that do not is that cues were often present (e.g., Ford, 1995; Hoch \& Tschirgi, 1983), and these may prompt the individual in such a way as to more accurately articulate his or her knowledge. In addition, online concurrent reports (e.g., Berry, 1983; Ford, 1995; Stenning \& van Lambalgen, 1999) are made in the presence of many task cues, which may also explain why they more accurately correspond to behavioral data than do retrospective reports.

Instead of appealing to functionally distinct systems, these findings can be interpreted as showing that participants vary as to the insight they have into their own reasoning, and with enough cuing a correspondence can be found. Such an interpretation does not deny that knowledge unavailable to conscious awareness is therefore unreportable, as described by Siegler and Stern (1998), but suggests that some studies that have demonstrated dissociations may not have exhausted all efforts to uncover what knowledge is possessed.

Tutoring studies, which include transfer tests, provide some scope for examining knowledge thought to be inaccessible to the reasoner. Early studies suggested that heuristic processing is insensitive to tutoring (e.g., Wason \& Golding, 1974; Wason \& Shapiro, 1971) and that erroneous responses result from preconscious and inflexible processes. Often in these examples, individuals returned to 
a default incorrect response strategy (e.g., Wason, 1969). Other demonstrations of differences between heuristic and analytic processing (e.g., inspection paradigm) provide evidence of differences in terms of speed of processing (e.g., Ball et al., 2003; Evans et al., 1987).

However, recent tutoring studies were able to facilitate correct reasoning, which transferred to variants of the original task (e.g., Fong \& Nisbett, 1991; Stenning \& van Lambalgen, 1999). These findings suggest that processes labeled implicit by dual theorists (e.g., Evans \& Over, 1996; Stanovich \& West, 2000) are accessible to the reasoner and can be modified. Moreover, studies directly contrasting implicit and explicit problem solving suggest that they cannot be reliably identified according to the differential demands made on working memory (e.g., P. J. Reber \& Kotovsky, 1997).

Dual-process theorists (i.e., Evans \& Over, 1996; Stanovich \& West, 2000) claim that implicit and automatic forms of reasoning are examples of System 1, and so both are characterized as inaccessible. However, there is evidence showing that automatic reasoning is analytic and accessible (e.g., Galotti et al., 1986), and that analytic reasoning is implicit and inaccessible to the reasoner (e.g., Siegler \& Stern, 1998). Both are examples of analytic processing and are inconsistent with the dualist proposal that heuristic processes are implicit. The DGC suggests that what distinguishes implicit and automatic reasoning is that for the latter, individuals can demonstrate metaknowledge, which can be reliably reproduced because the representations utilized are strong, stable, and distinctive, whereas in the former, individuals lack metaknowledge of their behavior, which cannot be reliably reproduced.

Clearly, further work is needed to disentangle differences between implicit and automatic forms of reasoning. One way of examining these distinctions might involve comparing practiced with novice reasoners by using a dual-task design. Typically, this design involves presenting two tasks simultaneously, one that makes high demands on attention (e.g., counting back in $3 \mathrm{sec}$ ) and a second problem-solving or reasoning task. One variant might involve repeating the task after a given interval (such as a day); if automatic and implicit knowledge is the same, both groups will reproduce the same responses, since implicit and automatic knowledge is highly inflexible. However, if there are differences between automatic and implicit knowledge, the novice group will not reliably reproduce the same response after an interval of time because they have developed unstable and indistinct representations, whereas the expert group will because they have formed strong and enduring representations. Furthermore, as an alternative to using verbal protocols as tests of awareness, participants could be presented with a specific problem state they had already experienced and be asked to predict the next move (this would measure the distinctiveness of their representations) or recall a sequence of moves made (this would measure the strength of their representations). Novices and experts should differ in their ability to accurately respond because their representations of the task differ according to whether they are implicit or automatic, whereas they should be equally poor if there is no distinction between "automatic" and "implicit" representations.

Neuroanatomical differences. Some dual-reasoning theorists (Evans \& Over, 1996; Stanovich \& West, 2000) claim that different neural pathways subserve two functionally distinct reasoning systems. There is, however, some disagreement as to which hemispheric region is associated with particular forms of reasoning. For example, clinical studies that examine patients with LH and RH damage (Golding, 1981) or that induce suppression of these regions (Deglin \& Kinsbourne, 1996) suggest that the LH is dominant in abstract deductive reasoning and that the RH is associated with content-based reasoning (e.g., Goel et al., 2000) and probabilistic inferencing (e.g., Wharton \& Grafman, 1998). Studies directly comparing probabilistic and deductive reasoning have found that there is a logic-specific network in both the $\mathrm{LH}$ and $\mathrm{RH}$ and that probabilistic reasoning activated mostly LH regions (Osherson et al., 1998; Parsons \& Osherson, 2001), which conflicts with other attempts to localize distinct reasoning mechanisms.

Neural localization of different reasoning mechanisms is not tantamount to evidence for qualitatively distinct reasoning systems (Sherry \& Schacter, 1987). Evidence from both neuroimaging and clinical studies suggests that different framings (e.g., familiar vs. unfamiliar context; concrete vs. abstract) of reasoning tasks such as syllogisms require functionally distinct forms of reasoning and are handled by distinct regions of the brain. It is important to ask whether the rules of operation differ or are the same for each distinct region. For example, Goel et al. (2000) found that there were no behavioral differences between response latencies for content and noncontentful syllogisms, and performance was also no different. However, there were distinct patterns of activation depending on the form that the syllogism took. Obviously, different representations are being formed when processing abstract and contentful material, but it is not clear how to distinguish the possibility that each region operates under a different and entirely separate reasoning system, and whether information is represented and stored differently but processed via a common reasoning mechanism.

\section{Conclusions}

The types of distinctions that dual-process theorists propose and the evidence that has been supportive of their claims have been evaluated in this review. Paralleling this, the review introduces the DGC framework, which describes how different types of reasoning come about through the graded properties of the representations that are generated while reasoning. These different approaches have been contrasted, and the review has sought to examine whether the types of processes identified by dualprocess theorists can be unified within a single-system framework that does not appeal to dissociable reasoning 
mechanisms. In so doing, it proposes that evidence used to support dual theories is consistent with single-system accounts. Moreover, the review suggests that certain types of reasoning phenomena have been misclassified by dual-process theorists as implicit, and should instead be classed as automatic reasoning. The grounds for making these claims arise from the DGC framework that assigns different functional roles to consciousness and not the reasoning system itself.

What the DGC is able to contribute to the reasoning field is that it permits organization and understanding of reasoning phenomena within a more plausible singlesystem framework for thinking about mental representation. Crucially, it highlights the ways in which implicit and automatic processing differ but also how they relate to each other and explicit processing under a single reasoning mechanism.

\section{REFERENCES}

AdLer, J. E. (1991). An optimist's pessimism: Conversation and conjunctions. In E. Eells \& T. Maruszewski (Eds.), Probability and rationality: Studies on L. Jonathan Cohen's philosophy of science (pp. 251-282). Amsterdam: Rodopi.

Agnoli, F., \& Krantz, D. H. (1989). Suppressing natural heuristics by formal instruction: The case of the conjunction fallacy. Cognitive Psychology, 21, 515-550.

ANDERSON, J. R. (1993). Rules of the mind. Hillsdale, NJ: Erlbaum.

ANDERSON, J. R. (1995). Learning and memory. New York: Wiley.

ANDERSON, J. R., \& BETZ, J. (2001). A hybrid model of categorization. Psychonomic Bulletin \& Review, 8, 629-647.

ANDERSON, J. R., \& Douglass, S. (201). Tower of Hanoi: Evidence for the cost of goal retrieval. Journal of Experimental Psychology: Learning, Memory, \& Cognition, 27, 1331-1346.

ANDERSON, J. R., \& SHEU, C. F. (1995). Causal inferences as perceptual judgments. Memory \& Cognition, 23, 510-524.

anderson, S. W., Bechara, A., Damasio, H., Tranel, D., \& Damasio, A. R. (1999). Impairment of social and moral behavior related to early damage in human prefrontal cortex. Nature Neuroscience, 2, 1032-1037.

Ball, L. J., Lucas, E. J., Miles, J. N. V., \& Gale, A. G. (2003). Inspection times and the selection task: What do eye-movements reveal about relevance effects? Quarterly Journal of Experimental Psychology, 56A, 1052-1077.

BARON, J. (1985). Rationality and intelligence. Cambridge: Cambridge University Press.

Benassi, V. A., \& KNOTH, R. L. (1993). The intractable conjunction fallacy: Statistical sophistication, instructional set, and training. Journal of Social Behavior \& Personality, 8, 83-96.

BERRY, D. (1983). Metacognitive experience and transfer of logical reasoning. Quarterly Journal of Experimental Psychology, 35A, 39-40.

Berry, D. (1991). The role of action in implicit learning. Quarterly Journal of Experimental Psychology, 43, 881-906.

BerRy, D., \& DienEs, Z. (1993). Implicit learning. Hove, U.K.: Erlbaum.

Betsch, T., \& Fiedler, K. (1999). Understanding conjunction effects in probability judgments: The role of implicit mental models. European Journal of Social Psychology, 29, 75-93.

BLOCK, N. (1995). On a confusion about a function of consciousness. Behavioral \& Brain Sciences, 18, 227-287.

Bourne, L. E. (1970). Knowing and using concepts. Psychological Review, 77, 546-556.

Braine, M. D. S., \& O'Brien, D. P. (1998). The theory of mental propositional logic: Description and illustration. In M. D. S. Braine \& D. P. O’Brien (Eds.), Mental logic (pp. 79-89). Mahwah, NJ: Erlbaum.

Brase, G. L., Cosmides, L., \& Tooby, J. (1998). Individuation, counting, and statistical inference: The role of frequency and whole-object representations in judgment under uncertainty. Journal of Experimental Psychology: General, 127, 3-21.
BuCCIARELLI, M. (2000). Reasoning strategies in syllogisms: Evidence for performance errors along with computational limitations. Behavioral \& Brain Sciences, 23, 669-670.

Bucciarelli, M., \& Johnson-Laird, P. N. (1999). Strategies in syllogistic reasoning. Cognitive Science, 23, 247-303.

Burns, B. D., \& VollmeYer, R. (2002). Goal specificity effects on hypothesis testing in problem solving. Quarterly Journal of Experimental Psychology, 55A, 241-261.

CeCI, S. J., \& LiKer, J. K. (1986). A day at the races: A study of IQ, expertise, and cognitive complexity. Journal of Experimental Psychology: General, 115, 255-266.

Cheesman, J., \& Merikle, P. M. (1984). Priming with and without awareness. Perception \& Psychophysics, 36, 387-395.

Cheng, P. W., \& Holyoak, K. J. (1985). Pragmatic schemas. Cognitive Psychology, 17, 391-416.

Cheng P. W., Holyoak, K. J., Nisbett, R. E., \& Oliver, L. M. (1986). Pragmatic versus syntactic approaches to training deductive reasoning. Cognitive Psychology, 18, 293-328.

Chrostowski, J. J., \& Griggs, R. A. (1985). The effects of problem content, instructions, and verbalization procedure on Wason's selection task. Current Psychological Research \& Reviews, 4, 99-107.

Cleeremans, A. (1997). Principles for implicit learning. In D. Berry (Ed.), How implicit is implicit learning? New York: Oxford University Press.

Cleeremans, A., Destrebecqz, A., \& Boyer, M. (1998). Implicit learning: News from the front. Trends in Cognitive Sciences, 2, 406-416.

Cleeremans, A., \& Jiménez, L. (2002). Implicit learning and consciousness: A graded, dynamical perspective. In R. M. French \& A. Cleeremans (Eds.), Implicit learning and consciousness: An empirical, philosophical and computational consensus in the making (pp. 1-40). Hove, U.K.: Psychology Press.

CoHen, L. J. (1981). Can human irrationality be experimentally demonstrated? Behavioral \& Brain Sciences, 4, 317-370.

Cosmides, L., \& Toовy, J. (1996). Are humans good intuitive statisticians after all? Rethinking some conclusions from the literature on judgment under uncertainty. Cognition, 58, 1-73.

Cosmides, L., \& Toовy, J. (2000). Consider the source: The evolution of adaptations for decoupling and metarepresentations. In D. Sperber (Ed.), Metarepresentation: A multidisciplinary perspective (pp. 53117). Oxford: Oxford University Press.

Crabb, B. T., \& Dark, V. J. (1999). Perceptual implicit memory requires attentional encoding. Memory \& Cognition, 27, 267-275.

Crandall, C. S., \& Greenfield, B. (1986). Understanding the conjunction fallacy: A conjunction of effects? Social Cognition, 4, 408-419.

Dawkins, R. (1976). The selfish gene. New York: Oxford University Press.

Deglin, V. L., \& Kinsbourne, M. (1996). Divergent thinking styles of the hemispheres: How syllogisms are solved during transitory hemisphere suppression. Brain \& Cognition, 31, 285-307.

Dienes, Z., \& FAHEY, R. (1998). The role of implicit memory in controlling a dynamic system. Quarterly Journal of Experimental Psychology, 51A, 593-614.

Dienes, Z., \& Perner, J. (2003). Unifying consciousness with explicit knowledge. In A. Cleeremans (Ed.), The unity of consciousness: Binding, integration, and dissociation (pp. 214-232). Oxford: Oxford University Press.

Dixon, J. A., \& Moore, C. F. (1997). Characterizing the intuitive representation in problem solving: Evidence from evaluating mathematical strategies. Memory \& Cognition, 25, 395-412.

Dudley, R. E. J., John, C. H., Young, A. W., \& Over, D. E. (1997). Normal and abnormal reasoning in people with delusions. British Journal of Clinical Psychology, 36, 243-258.

DulanY, D. E. (1991). Conscious representation and thought systems. In R. S. Wyer, Jr. \& T. K. Srull (Eds.), The content, structure, and operation of thought systems (pp. 97-120). Hillsdale, NJ: Erlbaum.

Dulany, D. E., \& Hilton, D. J. (1991). Conversational implicature, conscious representation, and the conjunction fallacy. Social Cognition, 9, 85-110.

Dunwoody, P. T., HaArbauer, E., Mahan, R. P., Marino, C., \& TAng, C. C. (2000). Cognitive adaptation and its consequences: A test of cognitive continuum theory. Journal of Behavioral Decision Making, $\underline{13}, \underline{35-54}$. 
EPSTEIN, S. (1973). The self-concept revisited: Or a theory of a theory. American Psychologist, 28, 404-416.

EPSTEIN, S. (1994). Integration of the cognitive and the psychodynamic unconscious. American Psychologist, 49, 709-724.

Epstein, S., Donovan, S., \& Denes-RaJ, V. (1999). The missing link in the paradox of the Linda conjunction problem: Beyond knowing and thinking of the conjunction rule, the intrinsic appeal of heuristic processing. Personality \& Social Psychology Bulletin, 25, 204-214.

Epstein, S., Pacini, R., Denes-Raj, V., \& Heier, H. (1996). Individual differences in intuitive-experiential and analytical-rational thinking styles. Journal of Personality \& Social Psychology, 71, 390-405.

Ericsson, K. A., Krampe, R. T., \& Tesch-Römer, C. (1993). The role of deliberate practice in the acquisition of expert performance. Psychological Review, 100, 363-406.

ERICSSON, K. A., \& SIMON, H. A. (1980). Verbal reports as data. Psychological Review, 87, 215-251.

EvANS, J. S. B. T. (1972). Interpretation and "matching bias" in a reasoning task. Quarterly Journal of Experimental Psychology, 24, 193199.

Evans, J. S. B. T. (1984). Heuristic and analytic processes in reasoning. British Journal of Psychology, 75, 451-468.

Evans, J. S. B. T. (1989). Biases in human reasoning: Causes and consequences. London: Erlbaum.

Evans, J. S. B. T. (1995). Relevance and reasoning. In S. E. Newstead \& J. S. B. T. Evans (Eds.), Perspectives on thinking and reasoning (pp. 147-172). Hove, U.K.: Erlbaum.

Evans, J. S. B. T. (1996). Deciding before you think: Relevance and reasoning in the selection task. British Journal of Psychology, 87, 223-240.

EvANS, J. S. B. T. (1998). Matching bias in conditional reasoning: Do we understand it after 25 years? Thinking \& Reasoning, 4, 45-82.

Evans, J. S. B. T. (2000). What could and could not be a strategy in reasoning. In W. Schaeken, D. DeVooght, \& A. D. D. Vandierendonck (Eds.), Deductive reasoning and strategies (pp. 1-22). Mahwah, NJ: Erlbaum.

Evans, J. S. B. T. (2002). Logical and human reasoning: An assessment of the deductive paradigm. Psychological Bulletin, 128, 978-996.

Evans, J. S. B. T., Ball, L. J., \& Brooks, P. G. (1987). Attention bias and decision order in a reasoning task. Quarterly Journal of Experimental Psychology, 51A, 811-814.

Evans, J. S. B. T., Barston, J. L., \& Pollard, P. (1983). On the conflict between logic and belief in syllogistic reasoning. Memory \& Cognition, 11, 295-306.

EVANS, J. S. B. T., \& OvER, D. E. (1996). Rationality and reasoning. Hove, U.K.: Psychology Press.

Evans, J. S. B. T., \& Pollard, P. (1990). Belief bias and problem complexity in deductive reasoning. In J.-P. Caverni, J.-M. Fabré, \& M. Gonzalez (Eds.), Cognitive biases (pp. 131-154). Amsterdam: Elsevier, North-Holland.

Evans, J. S. B. T., \& WAson, P. C. (1976). Rationalisation in a reasoning task. British Journal of Psychology, 63, 205-212.

FARAH, M. J. (1994). Visual perception and visual awareness after brain damage: A tutorial overview. In C. Umiltà \& M. Moscovitch (Eds.), Attention and performance $X V$ : Conscious and nonconscious information processing (pp. 37-76). Cambridge, MA: MIT Press.

Farah, M. J., Monheit, M. A., \& Wallace, M. A. (1991). Unconscious perception of "extinguished" visual stimuli: Reassessing the evidence. Neuropsychologia, 29, 949-958.

Farah, M. J., O'Reilly, R. C., \& Vecera, S. P. (1993). Dissociated overt and covert recognition as an emergent property of lesioned attractor networks. Psychological Review, 100, 571-588.

FidDICK, L., Cosmides, L., \& ToOBY, J. (2000). No interpretation without representation: The role of domain-specific representations and inferences in the Wason selection task. Cognition, 77, 1-79.

FIEDLER, K. (1988). The dependence of the conjunction fallacy on subtle linguistic factors. Psychological Research, 50, 123-129.

Fillenbaum, S. (1976). Inducements: On the phrasing and logic of conditional promises, threats, and warnings. Psychological Research, 38, 231-250.

FISK, J. E., \& PIDGEON, N. (1996). Component probabilities and the con- junction fallacy: Resolving signed summation and the low component model in a contingent approach. Acta Psychologica, 94, 1-20.

Fong, G. T., Krantz, D. H., \& Nisbett, R. E. (1986). The effects of statistical training on thinking about everyday problems. Cognitive Psychology, 18, 253-292.

FoNG, G. T., \& NISBETT, R. E. (1991). Immediate and delayed transfer of training effects in statistical reasoning. Journal of Experimental Psychology: General, 120, 34-45.

FoRD, M. (1995). Two modes of mental representation and problem solution in syllogistic reasoning. Cognition, 54, 1-71.

Freud, S. (1953). The interpretation of dreams. In J. Strachey (Ed. \& Trans.), The standard edition of the complete psychological works of Sigmund Freud (Vols. 4 and 5). London: Hogarth. (Original work published 1900)

Galotti, K. M., Baron, J., \& Sabini, J. P. (1986). Individual differences in syllogistic reasoning: Deduction rules or mental models? Journal of Experimental Psychology: General, 115, 16-25.

GedDes, B. W., \& Stevenson, R. J. (1997). Explicit learning of a dynamic system with a non-salient pattern. Quarterly Journal of Experimental Psychology, 50A, 742-765.

Gigerenzer, G., \& Hoffrage, U. (1995). How to improve Bayesian reasoning without instruction: Frequency formats. Psychological Review, 102, 684-704.

GILINSKY, A. S., \& JUDD, B. B. (1994). Working memory and bias in reasoning across the life span. Psychology \& Aging, 9, 356-371.

Goel, V. (1995). Sketches of thought. Cambridge, MA: MIT Press.

Goel, V., Buchel, C., Frith, C., \& Dolan, R. J. (2000). Dissociation of mechanisms underlying syllogistic reasoning. NeuroImage, $\underline{\mathbf{1 2}}_{2}$ 504-514.

GoEL, V., \& Dolan, R. J. (2001). Functional neuroanatomy of threeterm relational reasoning. Neuropsychologia, 39, 901-909.

Goel, V., Gold, B., Kapur, S., \& Houle, S. (1997). The seats of reason: A localization study of deductive $\&$ inductive reasoning using PET (O15) blood flow technique. NeuroReport, 8, 1305-1310.

Golding, E. (1981). The effect of unilateral brain lesion on reasoning. Cortex, 17, 31-40.

GoodwIn, R. Q., \& Wason, P. C. (1972). Degrees of insight. British Journal of Psychology, 63, 205-212.

Green, D. W., \& Larking, R. (1995). The locus of facilitation in the abstract selection task. Thinking \& Reasoning, 1, 183-199.

Griggs, R. A., \& Cox, J. R. (1982). The elusive thematic-materials effects in Wason's selection task. British Journal of Psychology, 73, 407-420.

Guilford, J. P. (1959). Three faces of intellect. American Psychologist, 14, 469-479.

Hammond, K. R. (1996). Human judgment and social policy. New York: Oxford University Press.

Hammond, K. R., \& Summers, D. A. (1972). Cognitive control. Psychological Review, 79, 58-67.

HaWkins, J., Pea, R. D., Glick, J., \& Scribner, S. (1984). "Merds that laugh don't like mushrooms": Evidence for deductive reasoning by preschoolers. Developmental Psychology, 20, 584-594.

HaYes, N., \& BroadBent, D. E. (1988). Two modes of learning for interactive tasks. Cognition, 28, 249-276.

Hertwig, R., Ortmann, A., \& Gigerenzer, G. (1997). Deductive competence: A desert devoid of content and context. Cahiers de Psychologie Cognitive, 16, 102-107.

Hilton, D. J. (1995). The social context of reasoning: Conversational inferences and rational judgment. Psychological Review, 118, 248271.

Hoch, S. J., \& Tschirgi, J. E. (1983). Cue redundancy and extra logical inferences in a deductive reasoning task. Memory \& Cognition, 11, 200-209.

Hoch, S. J., \& Tschirgi, J. E. (1985). Logical knowledge and cue redundancy in deductive reasoning. Memory \& Cognition, 13, 453-462.

Holender, D. (1986). Semantic activation without conscious activation in dichotic listening, parafoveal vision, and visual masking: A survey and appraisal. Behavioral \& Brain Sciences, 9, 1-66.

Holland, J. H., Holyoak, K. J., Nisbett, R. E., \& Thagard, P. R. (1989). Induction: Processes of inference, learning and discovery. Cambridge, MA: MIT Press. 
Houde, O., Zago, L., Crivello, F., Moutier, S., Pineau, A., Mazoyer, B., \& Tzourio-Mazoyer, N. (2001). Access to deductive logic depends on a right ventromedial prefrontal area devoted to emotion and feeling: Evidence from a training paradigm. NeuroImage, 14, 1486-1492.

HuQ, S. F., Garety, P. A., \& Hemsley, D. R. (1988). Probabilistic judgements in deluded and non-deluded subjects. Quarterly Journal of Experimental Psychology, 40A, 801-812.

JACOBY, L. L., LEVy, B. A., \& STEINBACH, K. (1992). Episodic transfer and automaticity: Integration of data-driven and conceptually driven processing in rereading. Journal of Experimental Psychology: Learning, Memory, \& Cognition, 18, 15-24.

JAMES, W. (1950). The principles of psychology. New York: Dover. (Original work published 1890)

Johnson-Laird, P. N., \& Byrne, R. M. J. (1991). Deduction. Hillsdale, NJ: Erlbaum.

Johnson-LaIRD, P. N., \& Wason, P. C. (1970). A theoretical analysis of insight into a reasoning task. Cognitive Psychology, 1, 134-148.

KAHNEMAN, D., \& Frederick, S. (2002). Representativeness revisited: Attribute substitution in intuitive judgment. In T. Gilovich, D. Griffin, \& D. Kahneman (Eds.), Heuristics and biases: The psychology of intuitive judgment. Cambridge: Cambridge University Press.

Karmiloff-Smith, A. (1986). From meta-processes to conscious access: Evidence from children's metalinguistic and repair data. Cognition, 23, 95-147.

KARNATH, H. O. (1988). Deficits in attention in acute and recovered visual hemi-neglect. Neuropsychologia, 26, 27-43.

Kemp, R., Chua, S., McKenna, P., \& DaVID, A. (1997). Reasoning and delusions. British Journal of Psychiatry, 170, 398-405.

KIRBY, K. N. (1994). Probabilities and utilities of fictional outcomes in Wason's four-card selection task. Cognition, 5, 1-28.

KLACZYNSKI, P. A. (2001). Analytical and heuristic processing influences on adolescent reasoning and decision making. Child Development, 72, 844-861.

KLACZYNSKI, P. A., Gelfand, H., \& ReEse, H. W. (1989). Transfer of conditional reasoning: Effects of explanations and initial problem types. Memory \& Cognition, 17, 208-220.

KlACZyNSKI, P. A., GoRDON, D. H., \& FAUTH, J. (1996). Goal-oriented reasoning and individual differences in critical reasoning biases. Journal of Educational Psychology, 89, 470-485.

Klayman, J., \& Ha, Y. W. (1987). Confirmation, disconfirmation and information in hypothesis testing. Psychological Review, 94, 211-228.

Knauff, M., Mulack, T., KassubeK, J., Salih, H. R., \& Greenlee, M. W. (2002). Spatial imagery in deductive reasoning: A functional MRI study. Cognitive Brain Research, 13, 203-212.

KoeHLER, J. J. (1996). The base rate fallacy reconsidered: Descriptive, normative and methodological challenges. Behavioral \& Brain Sciences, 19, 1-53.

Kunn, D. (2000). Metacognitive development. Current Directions in Psychological Science, 9, 178-181.

Kunimoto, C., Miller, J., \& Pashler, H. (2001). Confidence and accuracy of near-threshold discrimination responses. Consciousness \& Cognition, 10, 294-340.

LEVINSON, S. C. (1995). Interactional biases in human thinking. In E. Goody (Ed.), Social intelligence and interaction (pp. 221-260). Cambridge: Cambridge University Press.

MacDonald, P., \& MacLeod, C. M. (1998). The influence of attention at encoding on direct and indirect remembering. Acta Psychologica, 98, 298-310.

MankTelow, K. I., \& Over, D. E. (1995). Deontic reasoning. In S. E. Newstead \& J. S. B. T. Evans (Eds.), Perspectives on thinking and reasoning (pp. 91-114). Hove, U.K.: Erlbaum.

Margolis, H. (1987). Patterns, thinking, and cognition. Chicago: University of Chicago Press.

MARGOLIs, H. (2000). Wason's selection task with a reduced array [On line]. Psycoloquy, 11 (5).

Markovits, H., \& NANTEL, G. (1989). The belief-bias effect in the production and evaluation of logical conclusions. Memory \& Cognition, 17, 11-17.

MARKUS, H., \& ZAJONC, R. B. (1985). The cognitive perspective in so- cial psychology. In G. Lindzey \& E. Aronson (Eds.), Handbook of social psychology (3rd ed.). New York: Random House.

Mathis, W. D., \& Mozer, M. C. (1996, July). Conscious and unconscious perception: A computational theory. Paper presented at the 18th Annual Conference of the Cognitive Science Society, San Diego.

Meier, B., \& Perrig, W. J. (2000). Low reliability of perceptual priming: Consequences for the interpretation of functional dissociations between explicit and implicit memory. Quarterly Journal of Experimental Psychology, 53A, 211-233.

Morier, D. M., \& Borgida, E. (1984). The conjunction fallacy: A task specific phenomenon? Personality \& Social Psychology Bulletin, $\underline{\mathbf{1 0}}_{2}$ 243-252.

Moshman, D. (1999). Adolescent psychological development: Rationality, morality, and identity. Mahwah, NJ: Erlbaum.

Moshman, D. (2000). Diversity in reasoning and rationality: Metacognitive and developmental considerations. Behavioral \& Brain Sciences, 23, 689-690.

Newstead, S. E. (2000). Are there two different types of thinking? Behavioral \& Brain Sciences, 23, 690-691.

Newstead, S. E., Handley, S. J., Harley, C., Wright, H., \& FarRELLY, D. (2004). Individual differences in deductive reasoning. Quarterly Journal of Experimental Psychology, 57A, 33-60.

Newstead, S. E., Pollard, P., Evans, J. S. B. T., \& Allen, J. L. (1992). The source of belief bias effects in syllogistic reasoning. Cognition, 45, 257-284.

Newstead, S. E., Thompson, V. A., \& Handley, S. J. (2002). Generating alternatives: A key component in human reasoning? Memory \& Cognition, 30, 129-137.

Nisbett, R. E., KrantZ, D. H., Jepson, C., \& Kunda, Z. (1983). The use of statistical heuristics in everyday inductive reasoning. Psychological Review, 90, 339-363.

NisBeTt, R. E., \& Ross, L. (1980). Human inference: Strategies and shortcomings of social judgment. Englewood Cliffs, NJ: Prentice-Hall.

Nisbett, R. E., \& Wilson, T. D. (1977). Telling more than we can know: Verbal reports on mental processes. Psychological Review, $\mathbf{8 4}$, 231-259.

OAKHIll, J., Garnham, A., \& Johnson-Laird, P. N. (1990). Belief bias effects in syllogistic reasoning. In K. J. Gilhooly, M. T. G. Keane, R. H. Logie, \& G. Erdos (Eds.), Lines of thinking: Reflections on the psychology of thought (pp. 125-139). Chichester, U.K.: Wiley.

OAKHILL, J., \& JOHNSON-LAIRD, P. N. (1985). The effects of belief bias on the production of syllogistic conclusions. Quarterly Journal of Experimental Psychology, 37A, 553-569.

Oberauer, K. (2000). Do we need two systems for reasoning? Behavioral \& Brain Sciences, 23, 692-693.

O'Brien, G., \& OpIE, J. (1999). A connectionist theory of phenomenal experience. Behavioral \& Brain Sciences, 22, 127-196.

O'Reilly, R. C., \& Munakata, Y. (2000). Computational explorations in cognitive neuroscience: Understanding the mind by simulating the brain. Cambridge, MA: MIT Press.

Osherson, D., Perani, D., CapPa, S., Schnur, T., Grassi, F., \& Fazio, F. (1998). Distinct brain loci in deductive versus probabilistic reasoning. Neuropsychologia, 36, 369-376.

OsMAN, M. (2002, August). Is there evidence for unconscious reasoning processes? Proceedings of the 24th Annual Meeting of the Cognitive Science Society, Washington, DC.

Osman, M., \& LAming, D. (2001). Misinterpretation of conditional statements in Wason's selection task. Psychological Research, $\mathbf{6 5}$, 128-144.

Over, D., \& Evans, J. S. B. T. (1997). Two cheers for deductive competence. Current Psychology of Cognition, 16, 255-278.

PACINI, R., \& Epstein, S. (1999). The relation of rational and experiential processing styles to personality, basic beliefs, and the ratiobias phenomenon. Journal of Personality \& Social Psychology, 76, 972-987.

PARSONS, L. M., \& OSherson, D. (2001). New evidence for distinct right and left brain systems for deductive versus probabilistic reasoning. Cerebral Cortex, 11, 954-965.

Perruchet, P., \& Baveux, P. (1989). Correlational analyses of explicit and implicit memory performance. Memory \& Cognition, 17, 77-86. 
Perruchet, P., \& Vinter, A. (2002). The self-organising consciousness: A framework for implicit learning. In R. M. French \& A. Cleeremans (Eds.), Implicit learning and consciousness: An empirical, philosophical and computational consensus in the making (pp. 41-67). Hove, U.K.: Psychology Press.

Platt, R. D., \& GRIGGS, R.A. (1995). Facilitation and matching bias in the abstract selection task. Thinking \& Reasoning, 1, 55-77.

REBER, A. S. (1989). Implicit learning and tacit knowTedge. Journal of Experimental Psychology, 118, 219-235.

Reber, A. S. (1993). Implicit learning and tacit knowledge: An essay on the cognitive unconscious. New York: Oxford University Press.

REBER, P. J., \& KoTovsky, K. (1997). Implicit learning in problem solving: The role of working memory capacity. Journal of Experimental Psychology: General, 126, 178-203.

REEVES, T., \& LOCKHART, R. S. (1993). Distributed versus singular approaches to probability and errors in probabilistic reasoning. Journal of Experimental Psychology: General, 122, 207-226.

Reingold, E. M., Charness, N., Pomplun, M., \& Stampe, D. M. (2001). Visual span in expert chess players: Evidence from eye movements. Psychological Science, 12, 48-55.

Reingold, E. M., Charness, N., Schultetus, R. S., \& Stampe, D. M. (2001). Perceptual automaticity in expert chess players: Parallel encoding of chess relations. Psychonomic Bulletin \& Review, $\underline{\mathbf{8}, 504-}$ 510.

Reyna, V. F., \& Brainerd, C. J. (1990). Fuzzy processing in transitivity development. Annals of Operations Research, 23, 37-63.

RIPS, L. J. (1989). Similarity, typicality, and categorization. In S. Vosniadou \& A. Ortony (Eds.), Similarity and analogical reasoning (pp. 2159). Cambridge: Cambridge University Press.

Roberts, M. J. (1997). On dichotomies and deductive reasoning research. Current Psychology of Cognition, 16, 196-204.

RoBERTS, M. J. (1998). Inspection times and the selection task: Are they relevant? Quarterly Journal of Experimental Psychology, 51A, 781810 .

Roberts, M. J., \& Newton, E. J. (2001). Inspection times, the change task, and the rapid-response selection task. Quarterly Journal of Experimental Psychology, 54A, 1031-1048.

Roediger, H. L., III, \& McDermott, K. B. (1995). Creating false memories: Remembering words not presented in lists. Journal of Experimental Psychology: Learning, Memory, \& Cognition, 21, 803-814.

Rumelhart, D. E., \& McClelland, J. L. (1986). Parallel distributed processing: Explorations in the microstructure of cognition. Vol. 1: Foundations. Cambridge, MA: MIT Press.

Sá, W., West, R. F., \& Stanovich, K. (1999). The domain specificity and generality of belief bias: Searching for a generalizable critical thinking skill. Journal of Educational Psychology, 91, 497-510.

Schwarz, N. (1996). Cognition and communication: Judgmental biases, research methods, and the logic of conversation. Mahwah, NJ: Erlbaum.

Shafir, E., \& LeBoeuf, R. (2002). Rationality. Annual Review of Psychology, 53, 491-517.

SHANKS, D. R., \& CHANNON, S. (2002). Effects of a secondary task on "implicit" sequence learning: Learning or performance? Psychological Research, 66, 99-109.

SHANKs, D. R., \& Johnstone, T. (1999). Evaluating the relationship between explicit and implicit knowledge in a sequential reaction time task. Journal of Experimental Psychology: Learning, Memory, \& Cognition, 25, 1435-1451.

Shanks, D. R., \& St. John, M. F. (1994). Characteristics of dissociable human learning systems. Behavioral \& Brain Sciences, 17, $367-$ 447.

Sherry, D. F., \& Schacter, D. L. (1987). The evolution of multiple memory systems. Psychological Review, 94, 439-454.

Sides, A., Osherson, D., Bonini, N., \& ViaLe, R. (2002). On the reality of the conjunction fallacy. Memory \& Cognition, 30, 191-198.

SiEGLER, R. S., \& STERn, E. (1998). Conscious and unconscious strategy discoveries: A microgenetic analysis. Journal of Experimental Psychology: General, 127, 377-397.

SLOMAN, S. A. (1996). The empirical case for two systems of reasoning. Psychological Bulletin, 119, 3-22.
Sloman, S. A. (2002). Two systems of reasoning. In T. Gilovich, D. Griffin, \& D. Kahneman (Eds.), Heuristics and biases: The psychology of intuitive judgment (pp. 379-398). Cambridge: Cambridge University Press.

Sloman, S. A., Over, D., SlovaK, L., \& Stibel, J. M. (2003). Frequency illusions and other fallacies. Organizational Behavior \& Human Decision Processes, 91, 296-309.

Smith, E. E., \& Sloman, S. A. (1994). Similarity- versus rule-based categorization. Memory \& Cognition, 22, 377-386.

SMOLENSKY, P. (1988). On the proper treatment of connectionism. Behavioral \& Brain Sciences, 11, 1-23.

Sperber, D., Cara, F., \& Girotto,V. (1996). Relevance theory explains the selection task. Cognition, 57, 31-95.

Stanovich, K. E. (1999). Who is rational? Studies of individual differences in reasoning. Mahwah, $\mathrm{NJ}$ : Erlbaum.

Stanovich, K. E., \& West, R. F. (1998a). Individual differences in framing and conjunction effects. Thinking \& Reasoning, 4, 289-317.

StANOVICH, K. E., \& West, R. F. (1998b). Individual differences in rational thought. Journal of Experimental Psychology: General, 127, 161-188.

StANOVICH, K. E., \& West, R. F. (1998c). Who uses base rates and $\mathrm{P}(\mathrm{D} / \sim \mathrm{H})$ ? An analysis of individual differences. Memory \& Cognition, 26, 161-179.

STANOVICH, K. E., \& WEST, R. F. (2000). Individual differences in reasoning: Implications for the rationality debate? Behavioral \& Brain Sciences, 23, 645-665.

STANOVICH, K. E., \& WEST, R. F. (2002). Individual differences in reasoning: Implications for the rationality debate? In T. Gilovich, D. Griffin, \& D. Kahneman (Eds.), Heuristics and biases: The psychology of intuitive judgment (pp. 421-440). Cambridge: Cambridge University Press.

Stenning, K., \& van Lambalgen, M. (1999). Is psychology hard or impossible? Reflections on the conditional. In M. M. J. Gerbrandy, M. Marx, M. de Rijke, \& Y. Venema (Eds.), Liber amicorum for Johan van Benthem's 50th birthday (pp. 1-29). Amsterdam: Amsterdam University Press.

Stenning, K., \& Van Lambalgen, M. (2001). Semantics as a foundation for psychology: A case study of Wason's selection task. Journal of Logic, Language, \& Information, 10, 273-317.

Stevenson, R. J. (1997). Deductive reasoning and the distinction between implicit and explicit processes. Current Psychology of Cognition, 16, 222-229.

Stolarz-Fantino, S., Fantino, E., \& Kulik, J. (1996). The conjunction fallacy: Differential incidence as a function of descriptive frames and educational context. Contemporary Educational Psychology, 21, 208-218.

STROOP, J. (1935). Studies of inference in serial verbal reactions. Journal of Experimental Psychology, 18, 643-662.

Torrens, D., Thompson, V. A., \& CRAMER, K. M. (1999). Individual differences and the belief bias effect: Mental models, logical necessity, and abstract reasoning. Thinking \& Reasoning, 5, 1-28.

TVERSKY, A., \& Kahneman, D. (1974). Judgment under uncertainty: Heuristics and biases. Science, 185, 1124-1131.

TVERSKY, A., \& KAHNEMAN, D. (1983). Extensional versus intuitive reasoning: The conjunction fallacy in probability judgment. Psychological Review, 90, 293-315.

VELMANS, M. (1991). Is human information processing conscious? Behavioral \& Brain Sciences, 14, 651-726.

Volpe, D. T., LeDoux, J. E., \& Gazzaniga, M. S. (1979). Information processing of visual stimuli in an "extinguished" field. Nature, $\underline{\mathbf{2 8 2}}$, 722-724.

WALLACE, M. A., \& FARAH, M. J. (1992). Savings in relearning face-name associations as evidence for "covert recognition" in prosopagnosia. Journal of Cognitive Neuroscience, 4, 150-154.

WASON, P. C. (1968). Reasoning about a rule. Quarterly Journal of Experimental Psychology, 20, 273-281.

WASON, P. C. (1969). Regression in reasoning. British Journal of Psychology, 60, 471-480.

WASON, P. C., \& Evans, J. S. B. T. (1975). Dual processes in reasoning? Cognition, 3, 141-154. 
WASON, P. C., \& Golding, E. (1974). The language of inconsistency. British Journal of Psychology, 65, 537-546.

WASON, P. C., \& Johnson-LaIRD, P. N. (1970). A conflict between selecting and evaluating information in an inferential task. British Journal of Psychology, 61, 509-515.

WASON, P. C., \& SHAPIRO, D. (1971). Natural and contrived experience in a reasoning problem. Quarterly Journal of Experimental Psychology, 23, 63-71.
Wharton, C. M., \& Grafman, J. (1998). Deductive reasoning and the brain. Trends in Cognitive Sciences, 2, 54-59.

YATES, F. J., \& CARLSON, B. W. (1986). Conjunction errors: Evidence for multiple judgment procedures, including "signed summation." Organizational Behavior \& Human Decision Processes, 37, 230-253.

(Manuscript received August 8, 2003;

revision accepted for publication December 3, 2003.) 